\title{
Finite-time observer based tracking control of uncertain heterogeneous underwater vehicles using adaptive sliding mode approach
}

\section{Bo Chen}

UESTC: University of Electronic Science and Technology of China

\section{Yiyi Zhao}

SWUFE: Southwestern University of Finance and Economics

Jiangping Hu ( $\boldsymbol{\alpha}$ hj__lzu@163.com )

University of Electronic Science and Technology of China https://orcid.org/0000-0002-7559-8604

Bijoy Kumar Ghosh

TTU: Texas Tech University

\section{Research Article}

Keywords: Finite-time tracking control, underwater vehicle, uncertain heterogeneous dynamics, distributed observer, adaptive sliding mode control

Posted Date: April 8th, 2021

DOl: https://doi.org/10.21203/rs.3.rs-381021/v1

License: (9) This work is licensed under a Creative Commons Attribution 4.0 International License. Read Full License

Version of Record: A version of this preprint was published at Neurocomputing on January 1st, 2022. See the published version at https://doi.org/10.1016/j.neucom.2022.01.038. 


\title{
Finite-time observer based tracking control of uncertain heterogeneous underwater vehicles using adaptive sliding mode approach
}

\author{
Bo Chen · Yiyi Zhao · Jiangping Hu · Bijoy Kumar Ghosh
}

Received: date / Accepted: date

\begin{abstract}
In this study, we investigate a finite-time consensus tracking problem for a group of autonomous underwater vehicles (AUVs) with heterogeneous uncertain dynamics. We firstly propose a two-layer distributed control strategy, which consists of an upper-layer distributed observer and a lower-layer controller, without using any global information. Based on Hölder's inequality and the theory of finite-time stability, we develop a distributed finite-time observer for each follower to estimate the position information of a leader (i.e., an exosystem). Based on the sliding mode control method, we design a consensus tracking control scheme for each AUV, by which all follower AUVs can track the leader in finite-time. Secondly, when the parameters in the AUV dynamics are uncertain, we introduce a parameteradaptive sliding mode control algorithm to improve the control performance. Finally, simulation results are presented to demonstrate the effectiveness of the proposed control algorithms.
\end{abstract}

Keywords Finite-time tracking control $\cdot$ underwater vehicle $\cdot$ uncertain heterogeneous dynamics $\cdot$ distributed observer $\cdot$ adaptive sliding mode control

B. Chen · J. Hu

School of Automation Engineering, University of Electronic Science and Technology of China, Chengdu 611731, China

B. Chen

E-mail: cb_dzkjdx@std.uestc.edu.cn

J. $\mathrm{Hu}$

E-mail: hujp@uestc.edu.cn

Y. Zhao

School of Business Administration, Southwestern University of Finance and Economics, Chengdu 611130, China

E-mail: zhaoyy@swufe.edu.cn

B. K. Ghosh

Department of Mathematics and Statistics, Texas Tech University, Lubbock, TX, 79409-1042, USA

E-mail: bijoy.ghosh@ttu.edu

\section{Introduction}

In recent years distributed coordinate control of multiple autonomous underwater vehicles (AUVs) has attracted significant attention. This is because of the broad applications of multiple AUVs in many underwater missions, such as ocean graphic surveys, deep-sea inspections, seafloor mapping, and marine biology explorations [1-4]. Because of the complications of missions and some special performance requirements, the collaborative work of multiple relatively inexpensive and small AUVs has several advantages, such as better stability, greater efficiency, and extensive service areas [5-8], compared to an individual specialized AUV for performing solo missions. The distributed coordinate control of multiple AUVs requires that the control protocol for each AUV only uses local information to realize an overall goal. To this end, a series of coordination control approaches, such as neural networks [9-11], backstepping control [5, $12]$, adaptive control [6,13], and sliding mode control [1422], have been developed over the past decades to improve the coordination performance of multiple AUVs.

In contrast with other approaches, sliding mode control has some advantages, such as fast response, strong robustness, and simple physical implementation. Additionally, a sliding mode surface can be designed and is independent of the parameters and disturbances. Because of the complexity, unmodeled dynamics, uncertainties of AUV's models and environmental disturbances, Taha et al. [14] proposed a trajectory tracking control scheme based on a sliding mode control method to increase the model's robustness against bounded disturbances and also the lateral motion control of AUVs [15]. For the attitude control problem of AUVs with input nonlinearities and unknown disturbances, Cui et al. [16] simplified the three-dimensional (3D) space dynamics model of an AUV to second-order dynamics. An adaptive control law based on sliding mode control method was de- 
signed. Although the above control protocols based on sliding mode possessed satisfactory control effects, the systems showed a chattering phenomenon resulting from the discontinuous switching terms in the proposed protocols. To avoid chattering, Cui et al. [17] replaced the sign function with the saturation function. Zakeri et al. [18] used a sigmoid function to replace the sign function. However, these methods resulted in a reduction of tracking precision. Hence, $\mathrm{Li}$ and Zhu [19] proposed an adaptive high-order sliding mode control law based on a continuous adaptive function. However, the parameter uncertainties were not taken into consideration. Sun et al. [20] proposed a sliding mode control scheme combining a backstepping control technique. Parameter estimation was developed to address the problem associated with parameter uncertainties. Unfortunately, this controller was affected by the computational complexities of differential virtual control signals. Yang et al. [21] utilized a nonsingular fast terminal sliding mode control technique to solve a trajectory tracking control of AUVs when the attitude information cannot be obtained accurately and when the hydrodynamic parameters are uncertain. When the AUV systems suffered from ocean disturbances and uncertain parameters, Wang et al. [22] proposed an adaptive robust sliding-mode control scheme based on a neural network, dynamic surface, and backstepping technique.

Up to now, most of the aforementioned distributed coordinate control algorithms can achieve asymptotic stability or exponential stability for AUV systems. However the shorter stability time is crucial for distributed control of AUV systems. Thus finite-time distributed control algorithms are more desirable. Recently, Liu et al. [23] proposed a nonlinear finite-time sliding mode control strategy based on a disturbance observer to solve a trajectory tracking problem for underwater vehicles. Yang et al. [21] proposed a robust differentiator to estimate the external disturbances and uncertain parts in the linearly parameterized AUV model and then developed a finite-time controller based on the non-singular fast terminal sliding-mode control. Ali et al. [24] proposed a finite-time nonsingular fast terminal sliding mode control method based on the extended state observer to address the trajectory tracking control problem of an AUV subject to external disturbances and various hydrodynamic uncertainties. It is noted that the results mentioned above were obtained for a single AUV rather than multiple AUVs. The extension of finite-time control algorithms from single AUV to multiple AUVs is significant, especially in the case of leaderfollower systems with heterogeneous dynamics. When the control directions of multiple AUVs are not known, Gao and Guo [25] proposed a Nussbaum gain technique-based finite-time formation control approach to address a formation tracking problem. Qin et al. [26] presented a distributed finite-time containment control of multiple ocean bottom flying robots subject to model uncertainties, thruster faults, and external disturbances. Li et al. [27] proposed a nonsingular fast sliding mode control technique to address a finitetime consensus problem of multi-rigid systems by using the neighbors' acceleration information. Li and Wang [28] investigated a finite-time position consensus control problem of multiple AUVs by assuming that the parameters in the AUV model are precise, which may be inappropriate for the harsh underwater environment during application.

From the literature review and discussion mentioned above, even though there have been some research works on finitetime consensus control of multiple AUV systems by using the sliding mode control technique. However, accurate models of AUVs may not be applicable to cases with complex operation environment. Particularly, when the model parameters are uncertain and the complete state information cannot be available as well, the existing approaches are not available and thus a new control approach has to be investigated for uncertain heterogeneous AUV systems. For this purpose, this study considers a finite-time consensus control problem for an uncertain heterogeneous leader-follower AUV system by using a two-layer control strategy. The main contributions of this study are summarized as follows: First, we propose a finite-time distributed reduced-order observer to estimate the position of the leader. Second, we develop finite-time control laws for heterogeneous AUVs together with a novel sliding surface. Third, we present finite-time adaptive laws to estimate the uncertain parameters within the heterogenous AUV dynamics.

The remainder of this paper is organized as follows: Section 2 presents the preliminaries and problem formulation of the heterogeneous multiple AUVs. The main results of this study, including the distributed observer, the sliding-modebased and the adaptive sliding-mode-based finite-time consensus are presented in Sections 3, 4, and 5, respectively. The simulation studies are presented in Section 6, and Section 7 summarizes the study.

\section{Preliminaries and problem formulation}

This section presents some basic notations, the algebraic graph theory, some lemmas, and the problem formulation.

\subsection{Notations}

We denote $\operatorname{sig}^{\alpha}(x)=\operatorname{sgn}(x)|x|^{\alpha}$, where $\operatorname{sgn}(\cdot)$ represents the signum function and $x, \alpha \in R$. Note that $\frac{d|x|^{\alpha}}{d x}=\alpha \operatorname{sig}^{\alpha-1}(x)$. For vectors $\mathbf{x}=\left(x_{1}, x_{2}, \ldots, x_{n}\right)^{T}, \mathbf{y}=\left(y_{1}, y_{2}, \ldots, y_{n}\right)^{T} \in R^{n}$, and $\alpha \in R$, we denote $\mathbf{x}^{\alpha}=\left(x_{1}^{\alpha}, x_{2}^{\alpha}, \ldots, x_{n}^{\alpha}\right)^{T}, \operatorname{sig}^{\alpha}(\mathbf{x})=\left(\operatorname{sig}^{\alpha}\left(x_{1}\right)\right.$, $\left.\operatorname{sig}^{\alpha}\left(x_{2}\right), \ldots, \operatorname{sig}^{\alpha}\left(x_{n}\right)\right)^{T},|\mathbf{x}|^{\alpha}=\left(\left|x_{1}\right|^{\alpha},\left|x_{2}\right|^{\alpha}, \ldots,\left|x_{n}\right|^{\alpha}\right)$, especially $\operatorname{sgn}^{\alpha}(\mathbf{x})=\left(\operatorname{sgn}^{\alpha}\left(x_{1}\right), \operatorname{sgn}^{\alpha}\left(x_{2}\right), \ldots, \operatorname{sgn}^{\alpha}\left(x_{n}\right)\right)^{T} \cdot \mathbf{x} \cdot * \mathbf{y}$ denotes $\left(x_{1} y_{1}, x_{2} y_{2}, \ldots, x_{n} y_{n}\right)^{T} \in R^{n} .\|\mathbf{x}\|_{1}=\sum_{i=1}^{n}\left|x_{i}\right|,\|\mathbf{x}\|_{2}=$ 
$\sqrt{\mathbf{x}^{T} \mathbf{x}}$ denote the 1-norm and Euclidean norm of vector $\mathbf{x}$, respectively. A basic property is that $\|\mathbf{x}\|_{2} \leq\|\mathbf{x}\|_{1}, \forall \mathbf{x} \in R^{n}$. Let $I_{n}$ denote the $n \times n$ identity matrix. For arbitrary matrices $\mathbf{A} \in R^{m \times n}$ and $\mathbf{B} \in R^{p \times q}, \mathbf{A} \otimes \mathbf{B} \in R^{m p \times n q}$ denotes their Kronecker product, where $m, n, p, q$ are arbitrary positive integers. For any angle $\alpha \in R$, denote $s_{\alpha}=\sin \alpha, c_{\alpha}=\cos \alpha$.

\subsection{Algebraic Graph Theory}

In this study, we assume that there are $n$ follower AUVs. It is conventional to model the communication network associated with the group of AUVs using a directed graph (or digraph). Let $G=(V, E, A)$ be a weighted directed graph, where $V=\left\{\mathbb{v}_{1}, \mathbb{v}_{2}, \ldots, \mathbb{v}_{n}\right\}$ is the node set, $E=\left\{\left(\mathbb{v}_{j}, \mathbb{v}_{i}\right) \mid \mathbb{v}_{j}\right.$, $\left.\mathbb{v}_{i} \in V\right\}$ is the edge set, and $A=\left[a_{i j}\right] \in R^{n \times n}$ is a weighted adjacency matrix, where $a_{i j}>0$ if $\left(\mathbb{v}_{j}, \mathbb{v}_{i}\right) \in E$, otherwise $a_{i j}=0$. The set of in-neighbors of the agent $i$ is denoted by $N_{i}=\left\{j \in V \mid\left(\mathbb{v}_{j}, \mathbb{\mathbb { V }}_{i}\right) \in E\right\}$. Therefore, agent $i$ can receive the information of agent $j$ for $j \in N_{i}$. A directed path in a digraph $G$ is an edge sequence with distinct nodes. A directed tree is a directed graph, where every node has exactly one parent except for the root, and there exists a directional path from the root to the other nodes. A directed spanning tree of $G$ is a directed tree, where there exists a directional path from the root to the remaining nodes in $G$. A diagonal matrix $D=\operatorname{diag}\left\{d_{1}, d_{2}, \ldots, d_{n}\right\}$ is the degree matrix whose diagonal elements are defined by $d_{i}=\sum_{j \in N_{i}} a_{i j}$, and then the Laplacian matrix of the digraph $G$ is defined as $L=D-A$.

For the consensus tracking control of multiple AUVs, we assume that there exists a leader denoted by node $\mathbb{v}_{0}$, whose state is a time-varying reference signal for the followers denoted by nodes $\mathbb{v}_{1}, \mathbb{v}_{2}, \ldots, \mathbb{v}_{n}$. The communication topology graph of the leader-follower multiple AUVs is denoted as $\bar{G}=(\bar{V}, \bar{E})$, where $\bar{V}=V \cup\{0\}$ is the set of nodes, and $\bar{E} \subseteq$ $\bar{V} \times \bar{V}$. We use a diagonal matrix $B=\operatorname{diag}\left\{b_{1}, b_{2}, \ldots, b_{n}\right\}$ to describe the interaction relationships between the follower AUVs and the leader. If the $i$-th follower AUV has access to the leader, then $b_{i}>0$, otherwise $b_{i}=0$. Let $H=L+B$, then we have

Lemma 1 [29] The eigenvalues of the matrix $H$ have positive real parts if the augmented communication topology graph contains at least one directed spanning tree with root $\mathbb{v}_{0}$.

\subsection{Some Lemmas}

Some useful basic concepts and lemmas are reviewed in this section.

Consider the following nonlinear system

$\dot{\mathbf{x}}=\mathbf{f}(\mathbf{x}), \quad \mathbf{f}(\mathbf{0})=\mathbf{0}, \quad \mathbf{x} \in R^{n}$ where $\mathbf{f}(\mathbf{x}): U \rightarrow R^{n}$ is continuous on an open neighborhood $U$ of the origin $\mathbf{x}=\mathbf{0}$ in $R^{n}$.

Lemma 2 [30] For system (1), if there exists a positive definite Lyapunov function $V(\boldsymbol{x}): U_{0} \rightarrow R^{+}$, where $U_{0} \subseteq U \in R^{n}$ is an open neighborhood of the origin. For real number$s \quad>0$ and $\alpha \in(0,1)$ such that $\dot{V}(\boldsymbol{x})+c V^{\alpha}(\boldsymbol{x}) \leq 0, \boldsymbol{x} \in$ $U_{0} \backslash\{0\}$. Then, the origin of system (1) is finite-time stablility. If $U=U_{0}=R^{n}$, the origin is a globally finite-time stable equilibrium. Additionally, the finite settling time $T$ satisfies $T \leq \frac{V^{1-\alpha}(\boldsymbol{x}(\boldsymbol{\theta}))}{c(1-\alpha)}$.

Lemma 3 [31] For the nonlinear system (1), and suppose that there is a $C^{1}$ function $V(\boldsymbol{x}): D \rightarrow R$, where $D \in R^{n}$ is a neighborhood of the origin, and the real number $\lambda>0,0<$ $\gamma<1$ and $0<\eta<\infty$, if $V(\boldsymbol{x})$ with $V(\boldsymbol{0})=0$ is positive definite on $D$ and the following inequality holds:

$\dot{V}(\boldsymbol{x}) \leq-\lambda V^{\gamma}(\boldsymbol{x})+\eta$

Then, the trajectory of system (1) is practical finite-time stability.

Lemma 4 [32] Let $h_{i j}>0$ for $1 \leq i \leq n, 1 \leq j \leq m$ and $\sum_{j=1}^{m} p_{j}^{-1}=1$ with $p_{j}>1$ for $j=1,2, \ldots, m$. Then, the wellknown Hölder's inequality is expressed as follows:

$\sum_{i=1}^{n} \prod_{j=1}^{m} h_{i j} \leq \prod_{j=1}^{m}\left(\sum_{i=1}^{n} h_{i j}^{p_{j}}\right)^{\frac{1}{p_{j}}}$

and the special case Cauchy's inequality is expressed as follows:

$\left(\sum_{i=1}^{n} x_{i} y_{i}\right)^{2} \leq\left(\sum_{i=1}^{n} x_{i}^{2}\right)\left(\sum_{i=1}^{n} y_{i}^{2}\right)$

Lemma 5 [33] For any $x_{i} \in R, i=1,2, \ldots, n$, and a real number $q \in(0,1]$, the following inequality holds:

$$
\left(\sum_{i=1}^{n}\left|x_{i}\right|\right)^{q} \leq \sum_{i=1}^{n}\left|x_{i}\right|^{q} \leq n^{1-q}\left(\sum_{i=1}^{n}\left|x_{i}\right|\right)^{q}
$$

\subsection{Problem Formulation}

In this paper, we consider a system consisting of $n$ heterogenous AUVs, the kinematics and dynamics of the $i$ th AUV are described as follows [1]:

$\left\{\begin{array}{l}\dot{\mathbf{p}}_{i}=\mathbf{J}_{i}\left(\Theta_{i}\right) \mathbf{v}_{i} \\ \mathbf{M}_{i} \dot{\mathbf{v}}_{i}+\mathbf{D}_{i}\left(\mathbf{v}_{i}\right) \mathbf{v}_{i}+\mathbf{g}_{i}\left(\Theta_{i}\right)=\tau_{i}\end{array}\right.$

where $\mathbf{p}_{i}=\left(x_{i}, y_{i}, z_{i}\right)^{T}, \Theta_{i}=\left(\phi_{i}, \theta_{i}, \psi_{i}\right)^{T}$ denote the position and attitude vectors (i.e., roll $\phi_{i}$, pitch $\theta_{i}$, yaw $\psi_{i}$, as illustrated in Figure 1) in the inertial reference frame, respectively. $\mathbf{v}_{i}=\left(u_{i}, v_{i}, w_{i}\right)^{T}$ represents the velocity vector in the body- 


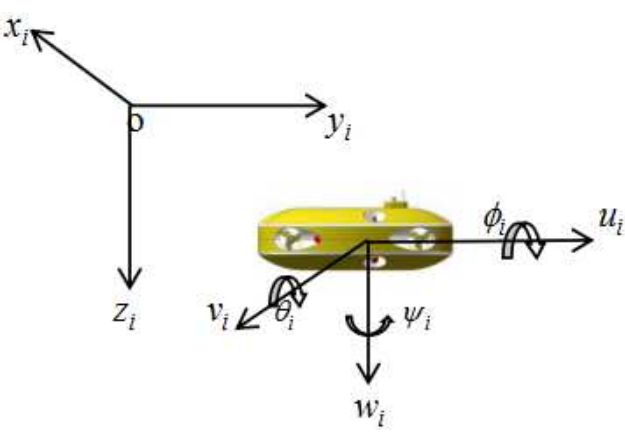

Fig. 1 The reference frames of AUV.

fixed reference frame, $\tau_{i}=\left(F_{u}, F_{v}, F_{w}\right)^{T}$ denotes the generalized thrust force, and $\mathbf{M}_{i}=\operatorname{diag}\left\{M_{\dot{u}_{i}}, M_{\dot{v}_{i}}, M_{\dot{w}_{i}}\right\}$ represents the inertia matrix, including the added mass. The transformation matrix $\mathbf{J}_{i}\left(\Theta_{i}\right)$ is then described as follows:

$\mathbf{J}_{i}\left(\Theta_{i}\right)=\left[\begin{array}{ccc}c_{\psi_{i}} c_{\theta_{i}}-s_{\psi_{i}} c_{\phi_{i}}+s_{\phi_{i}} s_{\theta_{i}} c_{\psi_{i}} & s_{\psi_{i}} s_{\phi_{i}}+s_{\theta_{i}} c_{\psi_{i}} c_{\phi_{i}} \\ s_{\psi_{i}} c_{\theta_{i}} & c_{\psi_{i}} c_{\phi_{i}}+s_{\phi_{i}} s_{\theta_{i}} s_{\psi_{i}} & -c_{\psi_{i}} s_{\phi_{i}}+s_{\theta_{i}} s_{\psi_{i}} c_{\phi_{i}} \\ -s_{\theta_{i}} & s_{\phi_{i}} c_{\theta_{i}} & c_{\phi_{i}} c_{\theta_{i}}\end{array}\right]$, $\mathbf{D}_{i}\left(\mathbf{v}_{i}\right)=\operatorname{diag}\left\{X_{u_{i}}, Y_{v_{i}}, Z_{w_{i}}\right\}+\operatorname{diag}\left\{D_{u}\left|u_{i}\right|, D_{v}\left|v_{i}\right|, D_{w}\left|w_{i}\right|\right\}$ is the damping matrix, where $X_{u_{i}}, Y_{v_{i}}$ and $Z_{w_{i}}$ represent the linear damping coefficients, $D_{u}, D_{v}$ and $D_{w}$ represent the nonlinear damping coefficients, $\mathbf{g}_{i}\left(\Theta_{i}\right)=\left[\left(W_{i}-B_{i}\right) \sin \theta_{i}\right.$, $\left.-\left(W_{i}-B_{i}\right) \cos \theta_{i} \sin \phi_{i},-\left(W_{i}-B_{i}\right) \cos \theta_{i} \cos \phi_{i}\right]^{T}$, where $W_{i}$ and $B_{i}$ represent the gravitational and buoyancy forces, respectively, is the restoring force. For brevity, we denote $\mathbf{J}_{i}=$ $\mathbf{J}_{i}\left(\Theta_{i}\right), \mathbf{D}_{i}=\mathbf{D}_{i}\left(\mathbf{v}_{i}\right), \mathbf{g}_{i}=\mathbf{g}_{i}\left(\Theta_{i}\right)$. Note that the transformation matrix $\mathbf{J}_{i}$ satisfies $\mathbf{J}_{i} \mathbf{J}_{i}^{T}=I_{3}$ [28].

Assumption 1 The inertia matrix $\boldsymbol{M}_{i}$ is a symmetric positive definite matrix.

In this study, we assume that the attitude angles of all follower AUVs are fixed. The dynamic equations of the $i$ th AUV can then be rewritten as follows:

$\left\{\begin{array}{l}\dot{\mathbf{p}}_{i}=\mathbf{J}_{i} \mathbf{v}_{i} \\ \mathbf{M}_{i} \dot{\mathbf{v}}_{i}+\mathbf{C}_{i} \mathbf{v}_{i}+\mathbf{D}_{i}\left|\mathbf{v}_{i}\right| \mathbf{v}_{i}+\mathbf{g}_{i}=\tau_{i}\end{array}\right.$

where $\mathbf{C}_{i}=\operatorname{diag}\left\{X_{u_{i}}, Y_{v_{i}}, Z_{w_{i}}\right\}, \mathbf{D}_{i}=\operatorname{diag}\left\{D_{u}, D_{v}, D_{w}\right\}$, and $\left|\mathbf{v}_{i}\right| \mathbf{v}_{i}=\left(\left|u_{i}\right| u_{i},\left|v_{i}\right| v_{i},\left|w_{i}\right| w_{i}\right)^{T}$. For the position trajectory tracking control problem, we consider the leader dynamics as follows to generate the tracking reference signal:

$\dot{\mathbf{x}}_{0}=A_{0} \mathbf{x}_{0}$

where $\mathbf{x}_{0}=\left(\mathbf{p}_{0}^{T}, \mathbf{v}_{0}^{T}\right)^{T}$ with $\mathbf{p}_{0} \in R^{3}$ and $\mathbf{v}_{0} \in R^{3}$ are the states of the leader, which are only available to a part of the follower-AUVs.

Some useful assumptions are given as follows:

Assumption 2 There exists a directed spanning tree with the leader node $\mathbb{v}_{0}$ as the root in the graph $\bar{G}$.

Assumption 3 For the leader, the states $\boldsymbol{p}_{0}$ and $\dot{\boldsymbol{p}}_{0}$ are all bounded. Without loss of generality, we assume $\left\|\dot{\boldsymbol{p}}_{0}\right\|_{\infty} \leq a_{0}$.
The main objective of the present study is to design a distributed consensus control law $\tau_{i}$, such that the states of all follower AUVs can track the time-varying reference trajectory in a finite time. In other words, there exists a constant $T_{0}$ such that the states of all AUVs satisfy $\mathbf{p}_{i}-\mathbf{p}_{0}=0$ and $\mathbf{v}_{i}-\mathbf{v}_{0}=0(i=1, \ldots, n)$ for $t \geq T_{0}$.

\section{Distributed Reduced-Order Observer}

This section presents an upper-layer distributed observer for each AUV before designing the tracking control. In this study, not all AUVs can obtained the state information of the leader directly, which requires the collaborative estimation of the state information of leader using the local information from the follower-AUVs. To this end, the following distributed reduced-order observer is designed for the $i$ th AUV to obtain an estimation of the leader's information $\mathbf{p}_{0}$ :

$$
\begin{aligned}
\dot{\hat{\mathbf{p}}}_{i 0}= & -l_{1} \operatorname{sig}^{\alpha}\left[\sum_{j=1}^{n} a_{i j}\left(\hat{\mathbf{p}}_{i 0}-\hat{\mathbf{p}}_{j 0}\right)+b_{i}\left(\hat{\mathbf{p}}_{i 0}-\mathbf{p}_{0}\right)\right] \\
& -l_{2} \operatorname{sgn}\left[\sum_{j=1}^{n} a_{i j}\left(\hat{\mathbf{p}}_{i 0}-\hat{\mathbf{p}}_{j 0}\right)+b_{i}\left(\hat{\mathbf{p}}_{i 0}-\mathbf{p}_{0}\right)\right]
\end{aligned}
$$

where $\hat{\mathbf{p}}_{i 0}$ denotes an estimation of $\mathbf{p}_{0}$, and $l_{1}>0, l_{2}>$ $a_{0}, 0<\alpha<1$.

To analyze the finite-time convergence of the distributed observer, we denote $\overline{\mathbf{p}}_{i 0}=\hat{\mathbf{p}}_{i 0}-\mathbf{p}_{0}, \dot{\mathbf{p}}_{L}=\mathbf{1}_{n} \otimes \dot{\mathbf{p}}_{0}, \overline{\mathbf{p}}=$ $\left(\overline{\mathbf{p}}_{i 0}^{T}, \ldots\right.$,

$\left.\overline{\mathbf{p}}_{n 0}^{T}\right)^{T}$ and $\xi=\left(H \otimes I_{3}\right) \overline{\mathbf{p}}$. The estimation error dynamics of the overall multiple AUV systems are expressed as follows:

$\dot{\overline{\mathbf{p}}}=-l_{1} \operatorname{sig}^{\alpha}(\xi)-l_{2} \operatorname{sgn}(\xi)-\dot{\mathbf{p}}_{L}$

Next, we will give the main result about the distributed observer (9).

Theorem 1 Under Assumptions 2 and 3, the estimation error system (10) is globally finite-time convergent, namely, there exists a constant $T_{01}$ such that $\hat{\boldsymbol{p}}_{i 0}=\boldsymbol{p}_{0}$ for $t \geq T_{01}$.

Proof Choose the Lyapunov candidate function as follows:

$V=\frac{1}{2} \overline{\mathbf{p}}^{T}\left(H \otimes I_{3}\right) \overline{\mathbf{p}}$

From Lemma 1, we have $H$ is positive, that is, $\lambda_{\max }(H)>$ $\lambda_{\text {min }}(H)>0$. Then, $V$ is positive definite and satisfies $V \leq$ $\frac{1}{2} \lambda_{\text {max }}(H)\|\overline{\mathbf{p}}\|_{2}^{2}$.

The time derivative of equation (11) is expressed as follows:

$\dot{V}=\xi^{T} \dot{\overline{\mathbf{p}}}=-l_{1} \xi^{T} \operatorname{sig}^{\alpha}(\xi)-l_{2} \xi^{T} \operatorname{sgn}(\xi)-\xi^{T} \dot{\mathbf{p}}_{L}$ 
From Lemma 4 , by taking $p_{1}=1+\alpha, p_{2}=\frac{1+\alpha}{\alpha}$, it follows that

$$
\begin{aligned}
\|\xi\|_{1} & =\sum_{i=1}^{3(n+1)}\left(\left|\xi_{i}\right| \times 1\right) \\
& \leq\left(\sum_{i=1}^{3(n+1)}\left|\xi_{i}\right|^{1+\alpha}\right)^{\frac{1}{1+\alpha}}\left(\sum_{i=1}^{3(n+1)} 1^{\frac{1+\alpha}{\alpha}}\right)^{\frac{\alpha}{1+\alpha}} \\
& =\left[\xi^{T} \operatorname{sig}^{\alpha}(\xi)\right]^{\frac{1}{1+\alpha}}[3(n+1)]^{\frac{\alpha}{1+\alpha}}
\end{aligned}
$$

and taking $p_{1}=1, p_{2}=+\infty$, we also have $\left|\xi^{T} \dot{\mathbf{p}}_{L}\right| \leq\left\|\xi^{T}\right\|_{1}$. $\left\|\dot{\mathbf{p}}_{L}\right\|_{\infty}$. Based on the two inequalities, from equation (12) with the fact that $\|\mathbf{x}\|_{2} \leq\|\mathbf{x}\|_{1}$, we have

$$
\begin{aligned}
\dot{V} \leq & -l_{1}(3 n)^{-\alpha}\|\xi\|_{2}^{1+\alpha}-\left(l_{2}-\left\|\dot{\mathbf{p}}_{L}\right\|_{\infty}\right)\|\xi\|_{2} \\
\leq & -l_{1}(3 n)^{-\alpha} \lambda_{\text {min }}^{1+\alpha}(H)\|\overline{\mathbf{p}}\|_{2}^{1+\alpha} \\
& -\left(l_{2}-\left\|\dot{\mathbf{p}}_{L}\right\|_{\infty}\right) \lambda_{\text {min }}(H)\|\overline{\mathbf{p}}\|_{2}
\end{aligned}
$$

Because $V \leq \frac{1}{2} \lambda_{\max }(H)\|\overline{\mathbf{p}}\|_{2}^{2}$, it can be obtained that

$$
\begin{array}{r}
\dot{V} \leq-\frac{l_{1}(3 n)^{-\alpha} \lambda_{\text {min }}^{1+\alpha}(H) 2^{\frac{1+\alpha}{2}}}{\lambda_{\text {max }}^{\frac{1+\alpha}{2}}(H)} V^{\frac{1+\alpha}{2}} \\
-\frac{\left(l_{2}-\left\|\dot{\mathbf{p}}_{L}\right\|_{\infty}\right) \lambda_{\text {min }}(H) \sqrt{2}}{\lambda_{\text {max }}^{\frac{1}{2}}(H)} V^{\frac{1}{2}}
\end{array}
$$

From inequality (15), Assumption 3 and Lemma 2, we can establish that $\hat{\mathbf{p}}_{i 0} \rightarrow \mathbf{p}_{0}$ in finite time. It is seen that each following AUV can adequately estimate the position of the leader through cooperative observation and estimation by using the distributed observer (9).

Remark 1 For the $i$ th follower AUVs, the observer (9) can estimate the leader's position information in finite-time, that is, there exists a constant $T_{01}$ satisfying

$T_{01} \leq \min \left\{\frac{(3 n)^{\alpha} \lambda_{\max }^{\frac{1+\alpha}{2}}(H) 2^{\frac{1-\alpha}{2}} V^{\frac{1-\alpha}{2}}(0)}{(1-\alpha) l_{1} \lambda_{\min }^{1+\alpha}(H)}, \frac{\sqrt{2} \lambda_{\max }^{\frac{1}{2}}(H) V^{\frac{1}{2}}(0)}{\left(l_{2}-\left\|\dot{\mathbf{p}}_{L}\right\|_{\infty}\right) \lambda_{\min }(H)}\right\}$. That is $\hat{\mathbf{p}}_{i 0}=\mathbf{p}_{0}$ for $t \geq T_{01}$.

\section{Finite-time Consensus Tracking}

To achieve the consensus tracking objective, now this section develops a lower-layer control strategy for each AUV. We firstly define a sliding mode surface for the $i$ th AUV as follows:

$\mathbf{S}_{i}=\dot{\varepsilon}_{i}+\lambda_{i} \operatorname{sig}^{\alpha_{1}}\left(\varepsilon_{i}\right)$

where $\varepsilon_{i}=\mathbf{p}_{i}-\hat{\mathbf{p}}_{i 0}, \lambda_{i}>0$ and $\alpha_{1} \in(0,1)$.

Next, we obtain the derivative of the sliding mode surface as follows:

$\dot{\mathbf{S}}_{i}=\mathbf{J}_{i} \dot{\mathbf{v}}_{i}-\ddot{\hat{\mathbf{p}}}_{i 0}+\lambda_{i} \alpha_{1}\left|\mathbf{p}_{i}-\hat{\mathbf{p}}_{i 0}\right|^{\alpha_{1}-1} \cdot *\left(\mathbf{J}_{i} \mathbf{v}_{i}-\dot{\hat{\mathbf{p}}}_{i 0}\right)$
The distributed finite-time control law for the $i$ th AUV can be designed as follows:

$$
\begin{aligned}
\tau_{i}=\mathbf{M}_{i} \mathbf{J}_{i}^{-1}[ & -\beta_{i} \operatorname{sig}^{\alpha_{2}}\left(\mathbf{S}_{i}\right)-\gamma_{i} \operatorname{sgn}\left(\mathbf{S}_{i}\right)+\mathbf{J}_{i} \mathbf{M}_{i}^{-1} \mathbf{C}_{i} \mathbf{v}_{i} \\
& +\mathbf{J}_{i} \mathbf{M}_{i}^{-1} \mathbf{D}_{i}\left|\mathbf{v}_{i}\right| \mathbf{v}_{i}+\mathbf{J}_{i} \mathbf{M}_{i}^{-1} \mathbf{g}_{i}+\ddot{\hat{\mathbf{p}}}_{i 0} \\
& \left.-\lambda_{i} \alpha_{1}\left|\mathbf{p}_{i}-\hat{\mathbf{p}}_{i 0}\right|^{\alpha_{1}-1} \cdot *\left(\mathbf{J}_{i} \mathbf{v}_{i}-\dot{\hat{\mathbf{p}}}_{i 0}\right)\right]
\end{aligned}
$$

where $\beta_{i}>0, \gamma_{i}>0$ and $\alpha_{2} \in(0,1)$.

Applying the controller (18) to equation (17) results in

$$
\dot{\mathbf{S}}_{i}=-\beta_{i} \operatorname{sig}^{\alpha_{2}}\left(\mathbf{S}_{i}\right)-\gamma_{i} \operatorname{sgn}\left(\mathbf{S}_{i}\right)
$$

Based on the above analysis, the tracking control performance of this controller and finite-time stability of the multiple AUV systems can be summarized as follows:

Theorem 2 Consider the multiple AUV systems (7). Under Assumption 2 and the proposed control law (18), the consensus tracking can then be achieved in finite-time for the multiple AUV systems.

Proof Choose the Lyapunov candidate function as $V(t)=$ $\sum_{i=1}^{n} V_{i}(t)$, where

$V_{i}=\frac{1}{2} \mathbf{S}_{i}^{T} \mathbf{S}_{i}$

From the definition of the Euclidean norm, we have $V$ and $V_{i}$ are positive definite and satisfy $V_{i}=\frac{1}{2}\left\|\mathbf{S}_{i}\right\|_{2}^{2}$.

The time derivative of $V$ is written as follows:

$\dot{V}=\sum_{i=1}^{n} \mathbf{S}_{i}^{T} \dot{\mathbf{S}}_{i}$

By substituting equation (19) into equation (21), we can obtain the following:

$$
\begin{aligned}
\dot{V} & =\sum_{i=1}^{n}\left[-\beta_{i} \mathbf{S}_{i}^{T} \operatorname{sig}^{\alpha_{2}}\left(\mathbf{S}_{i}\right)-\gamma_{i} \mathbf{S}_{i}^{T} \mathbf{s g n}\left(\mathbf{S}_{i}\right)\right] \\
& =\sum_{i=1}^{n}\left[-\beta_{i} \mathbf{S}_{i}^{T} \operatorname{sig}^{\alpha_{2}}\left(\mathbf{S}_{i}\right)-\gamma_{i}|| \mathbf{S}_{i} \|_{1}\right]
\end{aligned}
$$

From Lemma 4 , by taking $p_{1}=1+\alpha_{2}, p_{2}=\frac{1+\alpha_{2}}{\alpha_{2}}$, it follows that

$$
\begin{aligned}
\left\|\mathbf{S}_{i}\right\|_{1} & =\sum_{i=1}^{3}\left(\left|S_{i}\right| \times 1\right) \\
& \leq\left(\sum_{i=1}^{3}\left|S_{i}\right|^{1+\alpha_{2}}\right)^{\frac{1}{1+\alpha_{2}}}\left(\sum_{i=1}^{3} 1^{\frac{1+\alpha_{2}}{\alpha_{2}}}\right)^{\frac{\alpha_{2}}{1+\alpha_{2}}} \\
& =3^{\frac{\alpha_{2}}{1+\alpha_{2}}}\left(\mathbf{S}_{i}^{T} \operatorname{sig}^{\alpha_{2}}\left(\mathbf{S}_{i}\right)\right)^{\frac{1}{1+\alpha_{2}}}
\end{aligned}
$$

Then, we have $3^{-\alpha_{2}}\left\|\mathbf{S}_{i}\right\|_{1}^{1+\alpha_{2}} \leq \mathbf{S}_{i}^{T} \operatorname{sig}^{\alpha_{2}}\left(\mathbf{S}_{i}\right)$. From equation (22) with the fact that $\|\mathbf{x}\|_{2} \leq\|\mathbf{x}\|_{1}$, we can establish that

$\dot{V} \leq \sum_{i=1}^{n}\left(-3^{-\alpha_{2}} \beta_{i}\left\|\mathbf{S}_{i}\right\|_{2}^{1+\alpha_{2}}-\gamma_{i}\left\|\mathbf{S}_{i}\right\|_{2}\right)$ 
Because $V_{i}=\frac{1}{2}|| \mathbf{S}_{i} \|_{2}^{2}$, it can be established that

$\dot{V} \leq \sum_{i=1}^{n}\left(-3^{-\alpha_{2}} 2^{\frac{1+\alpha_{2}}{2}} \beta_{i} V_{i}^{\frac{1+\alpha_{2}}{2}}-\sqrt{2} \gamma_{i} V_{i}^{\frac{1}{2}}\right)$

From equation (19), we have

$\dot{V} \leq-3^{-\alpha_{2}} 2^{\frac{1+\alpha_{2}}{2}} \beta \sum_{i=1}^{n} V_{i}^{\frac{1+\alpha_{2}}{2}}-\sqrt{2} \gamma \sum_{i=1}^{n} V_{i}^{\frac{1}{2}}$

where $\beta=\min \left\{\beta_{1}, \beta_{2}, \ldots, \beta_{n}\right\}, \gamma=\min \left\{\gamma_{1}, \gamma_{2}, \ldots, \gamma_{n}\right\}$. Using Lemma 5, one can obtain the following:

$\dot{V} \leq-3^{-\alpha_{2}} 2^{\frac{1+\alpha_{2}}{2}} \beta\left(\sum_{i=1}^{n} V_{i}\right)^{\frac{1+\alpha_{2}}{2}}-\sqrt{2} \gamma\left(\sum_{i=1}^{n} V_{i}\right)^{\frac{1}{2}}$

From inequality (27) and Lemma 2, we can see that the position of all follower AUVs will converge to the sliding mode surface $\mathbf{S}_{i}=0$ in finite-time with upper time bound $T_{02}$ satisfying $T_{02} \leq \min \left\{\frac{3^{\alpha_{2}} \frac{1-\alpha_{2}}{2} V^{\frac{1-\alpha_{2}}{2}}(0)}{\beta\left(1-\alpha_{2}\right)}, \frac{\sqrt{2} V^{\frac{1}{2}}(0)}{\gamma}\right\}$.

Next, for the position error dynamics $\dot{\varepsilon}_{i}+\lambda_{i} \operatorname{sig}^{\alpha_{1}}\left(\varepsilon_{i}\right)$ $=0$, we can choose the Lyapunov candidate function as $V_{1}(t)=\sum_{i=1}^{n} V_{1 i}(t)$, where $V_{1 i}=\frac{1}{2} \varepsilon_{i}^{T} \varepsilon_{i}$. The time derivative of $V_{1}$ is expressed as follows:

$\dot{V}_{1}=\sum_{i=1}^{n} \varepsilon_{i}^{T} \dot{\varepsilon}_{i}=\sum_{i=1}^{n} \varepsilon_{i}^{T}\left(-\lambda_{i} \operatorname{sig}^{\alpha_{1}}\left(\varepsilon_{i}\right)\right)$

From Lemma 4, as in inequality (23), we have $3^{-\alpha_{1}}\left\|\varepsilon_{i}\right\|_{1}^{1+\alpha_{1}}$ $\leq \varepsilon_{i}^{T} \operatorname{sig}^{\alpha_{1}}\left(\varepsilon_{i}\right)$. With the fact $\|\mathbf{x}\|_{2} \leq\|\mathbf{x}\|_{1}$ and $V_{1 i}=\frac{1}{2}\left\|\varepsilon_{i}\right\|_{2}^{2}$, we can obtain the following:

$\dot{V}_{1} \leq \sum_{i=1}^{n}\left(-3^{-\alpha_{1}} \lambda_{i}\left\|\varepsilon_{i}\right\|_{2}^{1+\alpha_{1}}\right)=\sum_{i=1}^{n}\left(-3^{-\alpha_{1}} 2^{\frac{1+\alpha_{1}}{2}} \lambda_{i} V_{1 i}^{\frac{1+\alpha_{1}}{2}}\right)$

Using Lemma 5, one can obtain

$$
\begin{aligned}
\dot{V}_{1} & \leq-3^{-\alpha_{1}} 2^{\frac{1+\alpha_{1}}{2}} \lambda \sum_{i=1}^{n} V_{1 i}^{\frac{1+\alpha_{1}}{2}} \\
& \leq-(3)^{-\alpha_{1}} 2^{\frac{1+\alpha_{1}}{2}} \lambda\left(\sum_{i=1}^{n} V_{1 i}\right)^{\frac{1+\alpha_{1}}{2}} \\
& =-3^{-\alpha_{1}} 2^{\frac{1+\alpha_{1}}{2}} \lambda V_{1}^{\frac{1+\alpha_{1}}{2}}
\end{aligned}
$$

where $\lambda=\min \left\{\lambda_{1}, \lambda_{2}, \ldots, \lambda_{n}\right\}$.

From inequality (30) and Lemma 2, all errors converge

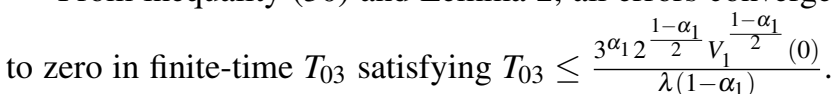
The position consensus of the leader-follower multiple AUVs is then achieved in finite-time with upper time bound $T_{0}=\max \left\{T_{01}, T_{02}, T_{03}\right\}$, which completes the proof.

\section{Adaptive Finite-time Consensus Tracking}

It is well known that during underwater tasks, because of the changing ocean environment, such as the flow rate, density, and temperature gradients of the water, the exact dynamic model parameters of AUVs may not be known a priori. Moreover, such uncertainties can limit the application of model-based controllers and affect the performance of the systems significantly. Therefore, it is necessary to design a control strategy that considers these uncertainties or eliminates them as much as possible. Motivated by this reason, adaptive finite-time position consensus tracking control will be developed in this section for multiple AUV systems where the parameters within the models have uncertainties.

We assume that the parameters $\mathbf{M}_{i}, \mathbf{C}_{i}, \mathbf{D}_{i}, \mathbf{g}_{i}$ within the AUV models are not known, then an adaptive sliding mode control strategy is designed for the $i$ th AUV as follows:

$$
\begin{aligned}
& \tau_{i}^{\prime}=\hat{\mathbf{M}}_{i}[-\beta_{i} \mathbf{J}_{i}^{-1} \operatorname{sig}^{\alpha_{2}}\left(\mathbf{S}_{i}\right)-\gamma_{i} \mathbf{J}_{i}^{-1} \operatorname{sgn}\left(\mathbf{S}_{i}\right)+\mathbf{J}_{i}^{-1} \ddot{\hat{\mathbf{p}}}_{i 0} \\
&\left.-\lambda_{i} \alpha_{1} \mathbf{J}_{i}^{-1}\left|\mathbf{p}_{i}-\hat{\mathbf{p}}_{i 0}\right|^{\alpha_{1}-1} \cdot *\left(\mathbf{J}_{i} \mathbf{v}_{i}-\dot{\hat{\mathbf{p}}}_{i 0}\right)\right] \\
&+\hat{\mathbf{C}}_{i} \mathbf{v}_{i}+\hat{\mathbf{D}}_{i}\left|\mathbf{v}_{i}\right| \mathbf{v}_{i}+\hat{\mathbf{g}}_{i} \\
&=\hat{\mathbf{M}}_{i} \mathbf{X}_{i}+\hat{\mathbf{C}}_{i} \mathbf{v}_{i}+\hat{\mathbf{D}}_{i}\left|\mathbf{v}_{i}\right| \mathbf{v}_{i}+\hat{\mathbf{g}}_{i}
\end{aligned}
$$

where $\mathbf{X}_{i}=-\beta_{i} \mathbf{J}_{i}^{-1} \operatorname{sig}^{\alpha_{2}}\left(\mathbf{S}_{i}\right)-\gamma_{i} \mathbf{J}_{i}^{-1} \operatorname{sgn}\left(\mathbf{S}_{i}\right)+\mathbf{J}_{i}^{-1} \ddot{\hat{\mathbf{p}}}_{i 0}-\lambda_{i} \alpha_{1}$ $\mathbf{J}_{i}^{-1}\left|\mathbf{p}_{i}-\hat{\mathbf{p}}_{i 0}\right|^{\alpha_{1}-1} . *\left(\mathbf{J}_{i} \mathbf{v}_{i}-\dot{\hat{\mathbf{p}}}_{i 0}\right), \hat{\mathbf{M}}_{i}=\operatorname{diag}\left\{\hat{M}_{i 1}, \hat{M}_{i 2}, \hat{M}_{i 3}\right\}$, $\hat{\mathbf{C}}_{i}=\operatorname{diag}\left\{\hat{C}_{i 1}, \hat{C}_{i 2}, \hat{C}_{i 3}\right\}, \hat{\mathbf{D}}_{i}=\operatorname{diag}\left\{\hat{D}_{i 1}, \hat{D}_{i 2}, \hat{D}_{i 3}\right\}, \hat{\mathbf{g}}_{i}=\left(\hat{g}_{i 1}\right.$, $\left.\hat{g}_{i 2}, \hat{g}_{i 3}\right)^{T}$. The according adaptive estimation laws are proposed as follows:

$$
\left\{\begin{array}{l}
\dot{\hat{M}}_{i j}=-k_{1 i j} \mathbf{S}_{i}^{T} \mathbf{J}_{i} \mathbf{X}_{i j} \\
\dot{\hat{C}}_{i j}=-k_{2 i j} \mathbf{S}_{i}^{T} \mathbf{J}_{i} \mathbf{M}_{i}^{-1} \mathbf{v}_{i j} \\
\dot{\hat{D}}_{i j}=-k_{3 i j} \mathbf{S}_{i}^{T} \mathbf{J}_{i} \mathbf{M}_{i}^{-1}\left|\mathbf{v}_{i j}\right| \mathbf{v}_{i j} \\
\dot{\hat{g}}_{i j}=-k_{4 i j} \mathbf{S}_{i}^{T} \mathbf{J}_{i} \mathbf{M}_{i}^{-1} \mathbf{1}_{i j}
\end{array}\right.
$$

where $\mathbf{X}_{i j}=\left(0, X_{i j}, 0\right)^{T}, \mathbf{v}_{i j}=\left(0, v_{i j}, 0\right)^{T},\left|\mathbf{v}_{i j}\right| \mathbf{v}_{i j}=\left(0,\left|v_{i j}\right| v_{i j}\right.$, $0)^{T}, \mathbf{1}_{i j}=\left(0,1_{i j}, 0\right)^{T}, j=1,2,3$ and $k_{1 i j}, k_{2 i j}, k_{3 i j}, k_{4 i j}$ are all positive gain parameters.

Theorem 3 Consider the multiple AUV systems (7). Under Assumption 2 and the control strategy (31), the consensus tracking can then be achieved in finite-time for the multiple AUV systems.

Proof Choose the Lyapunov candidate function as $V(t)=$ $\sum_{i=1}^{n} V_{i}(t)$, where

$$
\begin{aligned}
V_{i}= & \frac{1}{2} \mathbf{S}_{i}^{T} \mathbf{S}_{i}+\sum_{j=1}^{3} \frac{1}{2 k_{1 i j} M_{i j}} \tilde{M}_{i j}^{2}+\sum_{j=1}^{3} \frac{1}{2 k_{2 i j}} \tilde{C}_{i j}^{2} \\
& +\sum_{j=1}^{3} \frac{1}{2 k_{3 i j}} \tilde{D}_{i j}^{2}+\sum_{j=1}^{3} \frac{1}{2 k_{4 i j}} \tilde{g}_{i j}^{2},
\end{aligned}
$$

$\tilde{M}_{i j}=\hat{M}_{i j}-M_{i j}, \tilde{C}_{i j}=\hat{C}_{i j}-C_{i j}, \tilde{D}_{i j}=\hat{D}_{i j}-D_{i j}$ and $\tilde{g}_{i j}=$ $\hat{g}_{i j}-g_{i j}$ represent the estimation errors of parameters. We 
can also rewrite the Lyapunov candidate function as $V_{i}=$ $V_{i 1}+V_{i 2}$, where $V_{i 1}=\frac{1}{2} \mathbf{S}_{i}^{T} \mathbf{S}_{i}$ and $V_{i 2}=\sum_{j=1}^{3} \frac{1}{2 k_{1 i j} M_{i j}} \tilde{M}_{i j}^{2}+$ $\sum_{j=1}^{3} \frac{1}{2 k_{2 i j}} \tilde{C}_{i j}^{2}+\sum_{j=1}^{3} \frac{1}{2 k_{3 i j}} \tilde{D}_{i j}^{2}+\sum_{j=1}^{3} \frac{1}{2 k_{4 i j}} \tilde{g}_{i j}^{2}$. The time derivative of equation (33) is expressed as follows:

$$
\begin{gathered}
\dot{V}=\sum_{i=1}^{n}\left(\mathbf{S}_{i}^{T} \dot{\mathbf{S}}_{i}+\sum_{j=1}^{3} \frac{1}{k_{1 i j} M_{i j}} \tilde{M}_{i j} \dot{\hat{M}}_{i j}+\sum_{j=1}^{3} \frac{1}{2 k_{2 i j}} \tilde{C}_{i j} \dot{\hat{C}}_{i j}\right. \\
\left.+\sum_{j=1}^{3} \frac{1}{2 k_{3 i j}} \tilde{D}_{i j} \dot{\hat{\hat{D}}}_{i j}+\sum_{j=1}^{3} \frac{1}{2 k_{4 i j}} \tilde{g}_{i j} \dot{\hat{g}}_{i j}\right)
\end{gathered}
$$

By substituting equations (17) and (32) into equation (34), and from Assumption 1, we have

$$
\begin{aligned}
\dot{V}=\sum_{i=1}^{n} & \left(\mathbf { S } _ { i } ^ { T } \left(-\mathbf{J}_{i} \mathbf{M}_{i}^{-1} \mathbf{C}_{i} \mathbf{v}_{i}-\mathbf{J}_{i} \mathbf{M}_{i}^{-1} \mathbf{D}_{i}\left|\mathbf{v}_{i}\right| \mathbf{v}_{i}-\mathbf{J}_{i} \mathbf{M}_{i}^{-1} \mathbf{g}_{i}\right.\right. \\
& \left.+\mathbf{J}_{i} \mathbf{M}_{i}^{-1} \tau_{i}^{\prime}-\ddot{\hat{\mathbf{p}}}_{i 0}+\lambda_{i} \alpha_{1}\left|\mathbf{p}_{i}-\hat{\mathbf{p}}_{i 0}\right|^{\alpha_{1}-1} \cdot *\left(\mathbf{J}_{i} \mathbf{v}_{i}-\dot{\hat{\mathbf{p}}}_{i 0}\right)\right) \\
& -\sum_{j=1}^{3} \frac{\tilde{M}_{i j}}{k_{1 i j} M_{i j}} k_{1 i j} \mathbf{S}_{i}^{T} \mathbf{J}_{i} \mathbf{X}_{i j}-\sum_{j=1}^{3} \frac{1}{k_{2 i j}} \tilde{C}_{i j} k_{2 i j} \mathbf{S}_{i}^{T} \mathbf{J}_{i} \mathbf{M}_{i}^{-1} \mathbf{v}_{i j} \\
& -\sum_{j=1}^{3} \frac{1}{k_{3 i j}} \tilde{D}_{i j} k_{3 i j} \mathbf{S}_{i}^{T} \mathbf{J}_{i} \mathbf{M}_{i}^{-1}\left|\mathbf{v}_{i j}\right| \mathbf{v}_{i j} \\
& \left.-\sum_{j=1}^{3} \frac{1}{k_{4 i j}} \tilde{g}_{i j} k_{4 i j} \mathbf{S}_{i}^{T} \mathbf{J}_{i} \mathbf{M}_{i}^{-1} \mathbf{1}_{i j}\right)
\end{aligned}
$$

From the control strategy (31), we further have:

$$
\begin{aligned}
\dot{V}= & \sum_{i=1}^{n}\left(\mathbf { S } _ { i } ^ { T } \left(\mathbf{J}_{i} \mathbf{M}_{i}^{-1} \tilde{\mathbf{C}}_{i} \mathbf{v}_{i}+\mathbf{J}_{i} \mathbf{M}_{i}^{-1} \tilde{\mathbf{D}}_{i}\left|\mathbf{v}_{i}\right| \mathbf{v}_{i}+\mathbf{J}_{i} \mathbf{M}_{i}^{-1} \tilde{\mathbf{g}}_{i}\right.\right. \\
& \left.-\beta_{i} \mathbf{s i g}^{\alpha_{2}}\left(\mathbf{S}_{i}\right)-\gamma_{i} \mathbf{s g n}\left(\mathbf{S}_{i}\right)\right)-\sum_{j=1}^{3} \frac{1}{k_{2 i j}} \tilde{C}_{i j} k_{2 i j} \mathbf{S}_{i}^{T} \mathbf{J}_{i} \mathbf{M}_{i}^{-1} \mathbf{v}_{i j} \\
& -\sum_{j=1}^{3} \frac{1}{k_{3 i j}} \tilde{D}_{i j} k_{3 i j} \mathbf{S}_{i}^{T} \mathbf{J}_{i} \mathbf{M}_{i}^{-1}\left|\mathbf{v}_{i j}\right| \mathbf{v}_{i j} \\
& \left.-\sum_{j=1}^{3} \frac{1}{k_{4 i j}} \tilde{g}_{i j} k_{4 i j} \mathbf{S}_{i}^{T} \mathbf{J}_{i} \mathbf{M}_{i}^{-1} \mathbf{1}_{i j}\right)
\end{aligned}
$$

or equivalently,

$$
\begin{aligned}
\dot{V} & =\sum_{i=1}^{n}\left[-\beta_{i} \mathbf{S}_{i}^{T} \operatorname{sig}^{\alpha_{2}}\left(\mathbf{S}_{i}\right)-\gamma_{i} \mathbf{S}_{i}^{T} \mathbf{s g n}\left(\mathbf{S}_{i}\right)\right] \\
& =\sum_{i=1}^{n}\left[-\beta_{i} \mathbf{S}_{i}^{T} \operatorname{sig}^{\alpha_{2}}\left(\mathbf{S}_{i}\right)-\gamma_{i}|| \mathbf{S}_{i} \|_{1}\right] \leq 0
\end{aligned}
$$

From equation (37), we can show that $\mathbf{S}_{i}, \tilde{M}_{i j}, \tilde{C}_{i j}, \tilde{D}_{i j}$ and $\tilde{g}_{i j}$ are all bounded. Because $M_{i j}, k_{1 i j}, k_{2 i j}, k_{3 i j}$ and $k_{4 i j}$ are positive constants, the functions $V_{i 1}, V_{i 2}$ are bounded. From Lemma 4 and inequality (23), using the same analysis of Theorem 2, we can obtain the following:

$\dot{V} \leq \sum_{i=1}^{n}\left(-3^{-\alpha_{2}} \beta_{i}|| \mathbf{S}_{i}\left\|_{2}^{1+\alpha_{2}}-\gamma_{i}\right\| \mathbf{S}_{i} \|_{2}\right)$
Because $V_{i 1}=\frac{1}{2}\left\|\mathbf{S}_{i}\right\|_{2}^{2}$, it can be obtained that

$$
\begin{aligned}
\dot{V} & \leq \sum_{i=1}^{n}\left(-3^{-\alpha_{2}} 2^{\frac{1+\alpha_{2}}{2}} \beta_{i} V_{i 1}^{\frac{1+\alpha_{2}}{2}}-\sqrt{2} \gamma_{i} V_{i 1}^{\frac{1}{2}}\right) \\
& =\sum_{i=1}^{n}\left(-C_{1} V_{i 1}^{\frac{1+\alpha_{2}}{2}}-C_{2} V_{i 1}^{\frac{1}{2}}\right)
\end{aligned}
$$

where $C_{1}=3^{-\alpha_{2}} 2^{\frac{1+\alpha_{2}}{2}} \beta_{i}, C_{2}=\sqrt{2} \gamma_{i}$.

We can rewrite inequality (39) as follows:

$$
\begin{aligned}
& \dot{V} \leq \sum_{i=1}^{n}(-C_{1} V_{i 1}^{\frac{1+\alpha_{2}}{2}}-C_{1} V_{i 2}^{\frac{1+\alpha_{2}}{2}}+C_{1} V_{i 2}^{\frac{1+\alpha_{2}}{2}} \\
&\left.-C_{2} V_{i 1}^{\frac{1}{2}}-C_{2} V_{i 2}^{\frac{1}{2}}+C_{2} V_{i 2}^{\frac{1}{2}}\right) \\
&=\sum_{i=1}^{n}\left(-C_{1}\left(V_{i 1}^{\frac{1+\alpha_{2}}{2}}+V_{i 2}^{\frac{1+\alpha_{2}}{2}}\right)-C_{2}\left(V_{i 1}^{\frac{1}{2}}+V_{i 2}^{\frac{1}{2}}\right)\right. \\
&\left.+C_{1} V_{i 2}^{\frac{1+\alpha_{2}}{2}}+C_{2} V_{i 2}^{\frac{1}{2}}\right)
\end{aligned}
$$

From Lemma 5 and inequality (40), we obtain the following:

$$
\begin{aligned}
\dot{V} \leq & -C_{1} \sum_{i=1}^{n}\left(V_{i 1}+V_{i 2}\right)^{\frac{1+\alpha_{2}}{2}}-C_{2} \sum_{i=1}^{n}\left(V_{i 1}+V_{i 2}\right)^{\frac{1}{2}} \\
& +\sum_{i=1}^{n}\left(C_{1} V_{i 2}^{\frac{1+\alpha_{2}}{2}}+C_{2} V_{i 2}^{\frac{1}{2}}\right)
\end{aligned}
$$

From inequality (37), since $V_{i 2}$ is bounded, then $\sum_{i=1}^{n}\left(C_{1} V_{i 2}^{\frac{1+\alpha_{2}}{2}}+C_{2} V_{i 2}^{\frac{1}{2}}\right)$ is bounded. Let $\eta=\sup _{0<t<\infty}$ $\left\{\sum_{i=1}^{n}\left(C_{1} V_{i 2}^{\frac{1+\alpha_{2}}{2}}+C_{2} V_{i 2}^{\frac{1}{2}}\right)\right\}$, we obtain $\eta$ is a positive constant. Then, we further have

$$
\begin{aligned}
\dot{V} & \leq-C_{1}\left(\sum_{i=1}^{n} V_{i}\right)^{\frac{1+\alpha_{2}}{2}}-C_{2}\left(\sum_{i=1}^{n} V_{i}\right)^{\frac{1}{2}}+\eta \\
& =-C_{1} V^{\frac{1+\alpha_{2}}{2}}-C_{2} V^{\frac{1}{2}}+\eta
\end{aligned}
$$

From inequality (42) and Lemma 3, we can see that the sliding mode surface $\mathbf{S}_{i}$ can converge to zero in finite-time. Using a similar analysis of Theorem 2, the consensus tracking can be achieved for the multiple AUV systems in finitetime. This completes the proof.

Remark 2 Compared to the controller (18), the adaptive laws in the control strategy $\tau_{i}^{\prime}$ given by (31) can estimate the unknown parameters online. Therefore, the controller (31) is more practical for AUVs in real cases. Additionally, the sliding surface can converge to zero in finite-time with an upper bound $T_{02}$ while the observer (9) has the same time bound, as shown in Section 4. Thus the controller (31) can guarantee the finite-time convergence of the multiple AUV systems with upper time bound smaller than $T_{0}$. 


\section{Numerical Simulations}

In this section, we present two simulation examples to validate the dynamic performance of the two distributed consensus control strategies proposed in Sections 4 and 5, respectively.

Example 6.1: Consider a group of four follower AUVs and a leader. The interconnection network of the AUVs and the leader is illustrated in Fig.2.

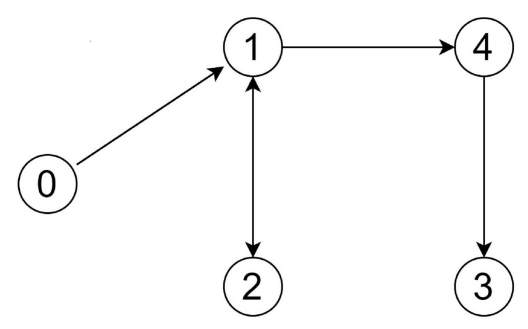

Fig. 2 Interconnection network topology

Without loss of generality, we assume that $a_{i j}=1$ if $j \in N_{i}$, otherwise $a_{i j}=0$. In the network, there is a directed spanning tree with the leader node $\mathbb{v}_{0}$ as the root. Thus the matrices $L$ and $H=L+B$ are expressed as follows:

$L=\left(\begin{array}{cccc}1 & -1 & 0 & 0 \\ -1 & 1 & 0 & 0 \\ 0 & 0 & 1 & -1 \\ -1 & 0 & 0 & 1\end{array}\right), H=\left(\begin{array}{cccc}2 & -1 & 0 & 0 \\ -1 & 1 & 0 & 0 \\ 0 & 0 & 1 & -1 \\ -1 & 0 & 0 & 1\end{array}\right)$

The model parameters are chosen as $\mathbf{M}_{i}=\operatorname{diag}\{2000$, $1800,1900\}, \mathbf{C}_{i}=\operatorname{diag}\{2,1,3\}, \mathbf{D}_{i}=\operatorname{diag}\{2,3,1\}, W_{i}=$ $1028 N, B_{i}=1008 N, \phi_{i}=\pi / 10, \theta_{i}=-\pi / 10$, and $\psi_{i}=\pi / 12$, $\forall i \in 1,2,3,4$. The leader dynamics is given as follows:

$$
\begin{aligned}
& \left(\begin{array}{c}
\dot{\mathbf{p}}_{0} \\
\dot{\mathbf{v}}_{0}
\end{array}\right)=\left(\begin{array}{cccccc} 
& & & 1 & 0 & 0 \\
\mathbf{0} & & 0 & -1 & 0 \\
& & & 0 & 0 & 1 \\
-1 & 0 & 0 & & & \\
0 & 1 & 0 & & \mathbf{0} \\
0 & 0 & -1 & &
\end{array}\right)\left(\begin{array}{l}
\mathbf{p}_{0} \\
\mathbf{v}_{0}
\end{array}\right) \\
& \left(\begin{array}{l}
\mathbf{p}_{0}(\mathbf{0}) \\
\mathbf{v}_{0}(\mathbf{0})
\end{array}\right)=\left(\begin{array}{llllll}
25 & 20 & -25 & 1.5 & 2 & -1.5
\end{array}\right)^{T}
\end{aligned}
$$

Choose the control law parameters as $\alpha=\alpha_{2}=0.9, \alpha_{1}=$ $0.95, l_{1}=2, l_{2}=26, \lambda=\operatorname{diag}\{15,9,9,6\}, \beta=\operatorname{diag}\{15,9,6$, $6\}, \gamma=\operatorname{diag}\{0.5,0.5,0.4,0.5\}$. The initial states of all AUVs are given as follows: $\mathbf{p}_{1}(\mathbf{0})=(25 ;-10 ; 25), \mathbf{v}_{1}(\mathbf{0})=(0 ; 0 ; 0)$ $\mathbf{p}_{2}(\mathbf{0})=(-25 ; 30 ; 25), \mathbf{v}_{2}(\mathbf{0})=(0 ; 0 ; 0), \mathbf{p}_{3}(\mathbf{0})=(25 ; 40 ;-25)$, $\mathbf{v}_{3}(\mathbf{0})=(0 ; 0 ; 0), \mathbf{p}_{4}(\mathbf{0})=(-20 ;-25 ; 20), \mathbf{v}_{4}(\mathbf{0})=(0 ; 0 ; 0)$.

The simulation results are shown in Fig. 3-5. The estimation performance is illustrated in Fig. 3, which indicates that the estimates $\hat{\mathbf{p}}_{i 0}$ equal the position state $\mathbf{p}_{0}$ of the leader after time 0.8 . The evolution of the sliding-mode surface

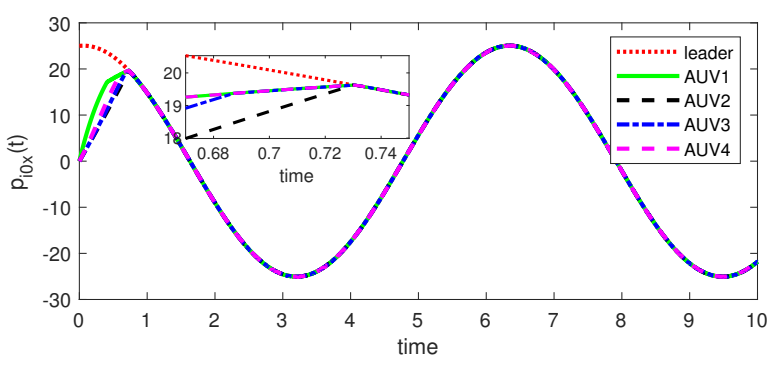

(a)

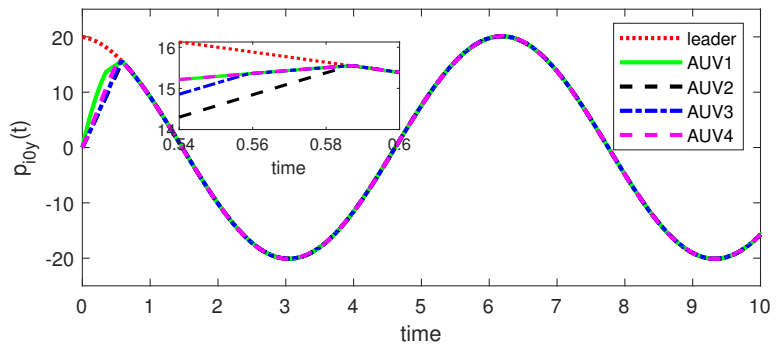

(b)

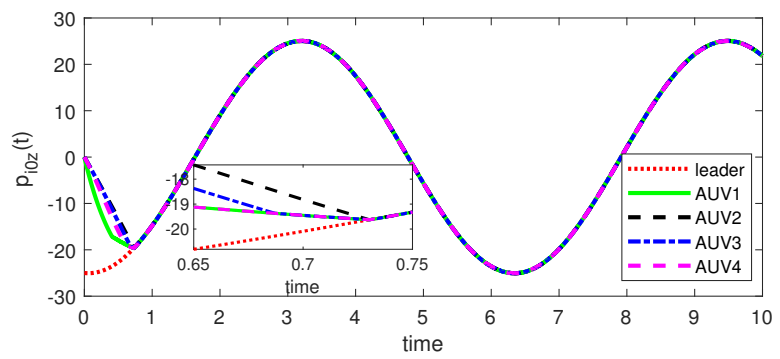

(c)

Fig. 3 The evolution of the state estimation of the distributed observer (9). (a) $x_{\hat{\mathbf{p}}_{i 0}} \rightarrow x_{\mathbf{p}_{0}}$; (b) $y_{\hat{\mathbf{p}}_{i 0}} \rightarrow y_{\mathbf{p}_{0}}$; (c) $z_{\hat{\mathbf{p}}_{i 0}} \rightarrow z_{\mathbf{p}_{0}}$.

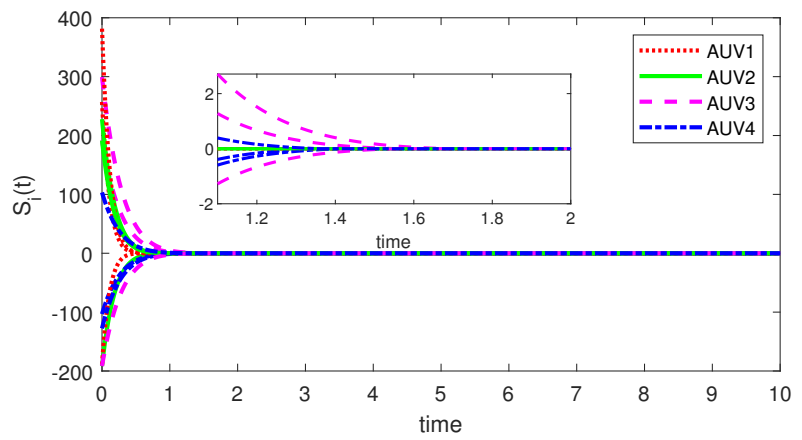

Fig. 4 The evolution of the sliding mode surface (19).

, is shown in Fig. 4. The position evolution of the AUVs is shown in Fig. 5, which demonstrates that the follower AUVs can follow the leader in finite-time under the proposed control strategy (18). Thus the simulation results validate Theorem 2.

Example 6.2: In this example, the interconnection topology is similar to that in Example 6.1. We assume that the 


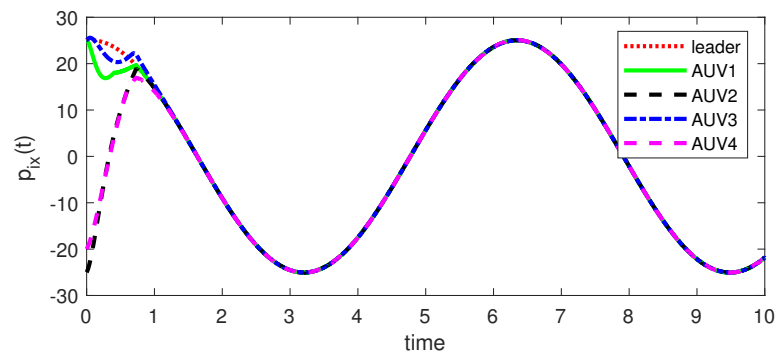

(a)

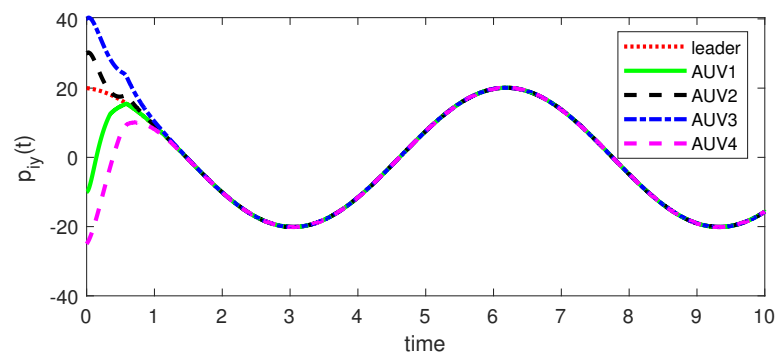

(b)

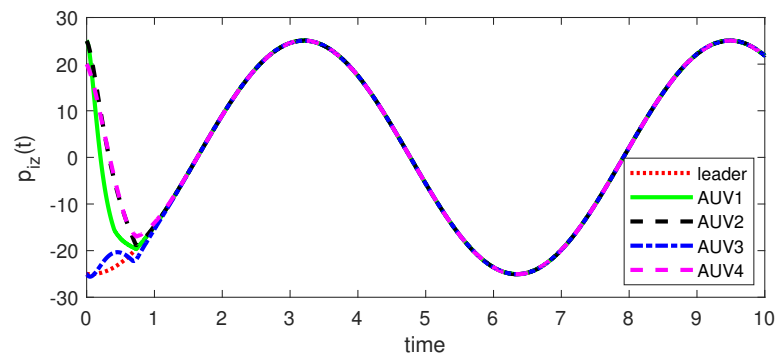

(c)

Fig. 5 The position evolution under the control strategy (18). (a) $x_{i} \rightarrow$ $x_{0}$; (b) $y_{i} \rightarrow y_{0}$; (c) $z_{i} \rightarrow z_{0}$.

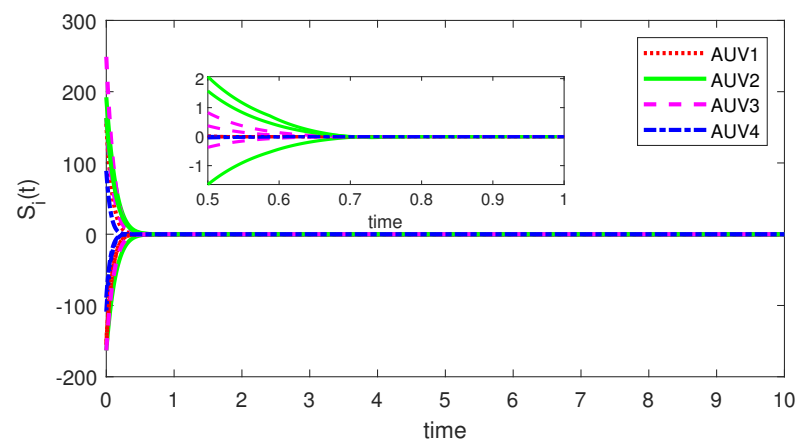

Fig. 6 The evolution of the adaptive sliding mode surface under the controller (31).

model parameters are unknown and employ the adaptive sliding mode control strategy (31) together with the adaptive law (32). We choose the control law parameter values as $\alpha=$ $\alpha_{1}=\alpha_{2}=0.9, l_{1}=2, l_{2}=26, \lambda=\operatorname{diag}\{12,9,9,6\}, \beta=$ $\operatorname{diag}\{18,12,15,24\}, \gamma=\operatorname{diag}\{0.5,0.5,0.4,0.5\}, k_{1 i j}=0.0015$ $k_{2 i j}=0.02, k_{3 i j}=0.02, k_{4 i j}=0.02$. We take the parameter

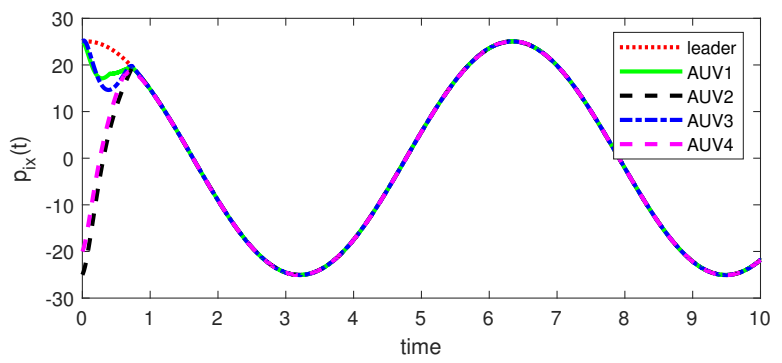

(a)

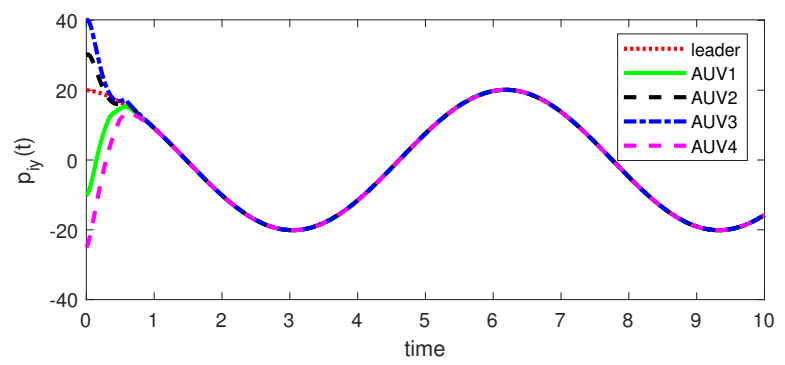

(b)

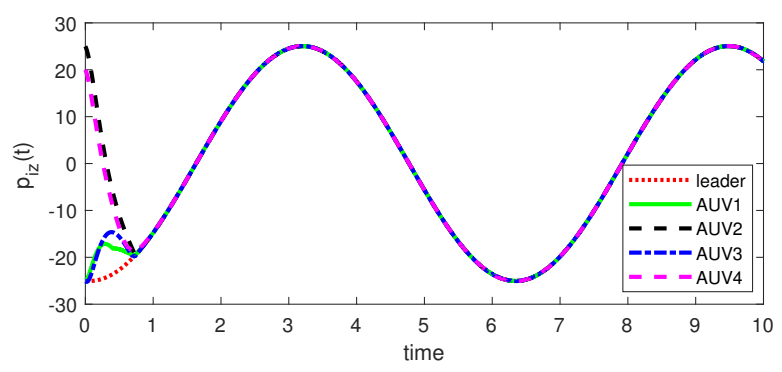

(c)

Fig. 7 The position evolution under the adaptive sliding mode control (31). (a) $x_{i} \rightarrow x_{0}$; (b) $y_{i} \rightarrow y_{0}$; (c) $z_{i} \rightarrow z_{0}$.

values in Example 6.1 as the initial values of the adaptation law (32), i.e., $\hat{\mathbf{M}}_{i}(\mathbf{0})=\operatorname{diag}\{2000,1800,1900\}, \hat{\mathbf{C}}_{i}(\mathbf{0})=\operatorname{diag}\{2,1,3\}$, $\hat{\mathbf{D}}_{i}(\mathbf{0})=\operatorname{diag}\{2,3,1\}, \hat{\mathbf{g}}_{i}(\mathbf{0})=\operatorname{diag}\{-6.1803,-5.8779,-18.0902\}, \forall i \in$ $1,2,3,4$. The initial states of all follower AUVs are similar to those in Example 6.1. The simulation results are shown in Fig. 6-8.

Fig. 6 shows the evolution of the adaptive sliding-mode surface under the controller (31). The AUVs' position evolution under the adaptive sliding mode control (31) is depicted in Fig. 7, which shows that the AUVs can follow the leader in a shorter time than in Example 6.1. The evolution of the parameter estimation errors is shown in Fig. 8. The simulation results validate Theorem 3 when the parameters within the AUV models are unknown.

\section{Conclusion}

In this study, we have investigated a finite-time consensus tracking problem for multiple AUV systems with uncertain 


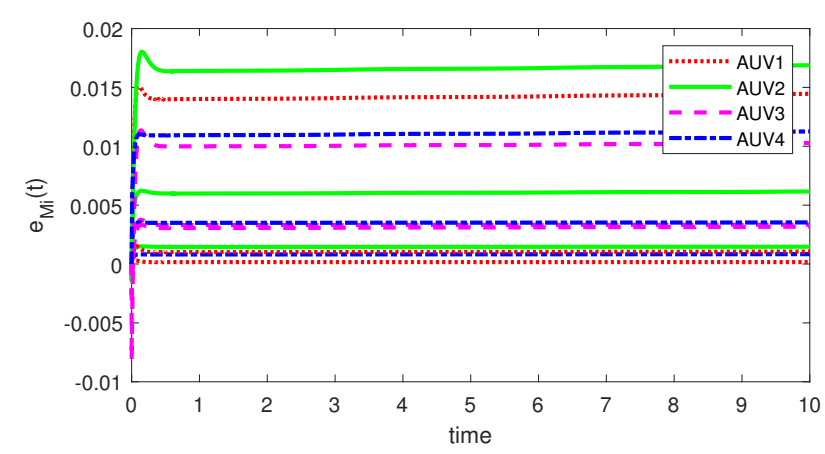

(a)

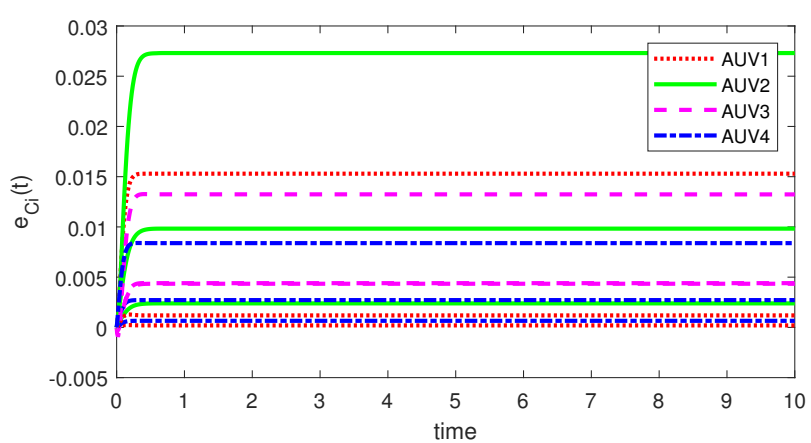

(b)

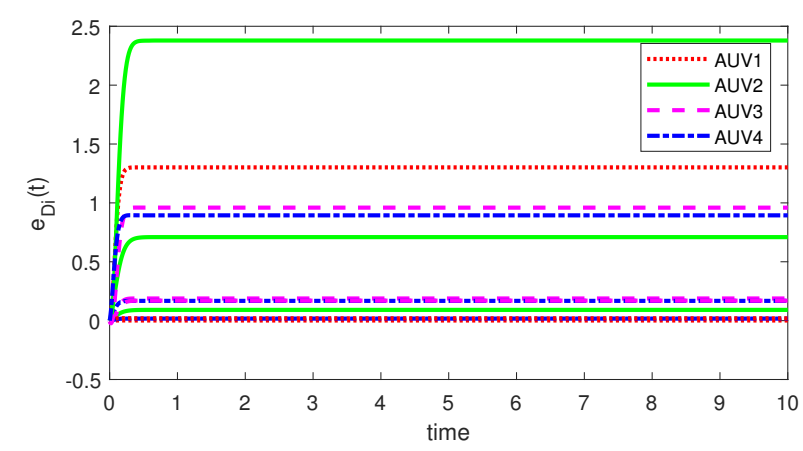

(c)

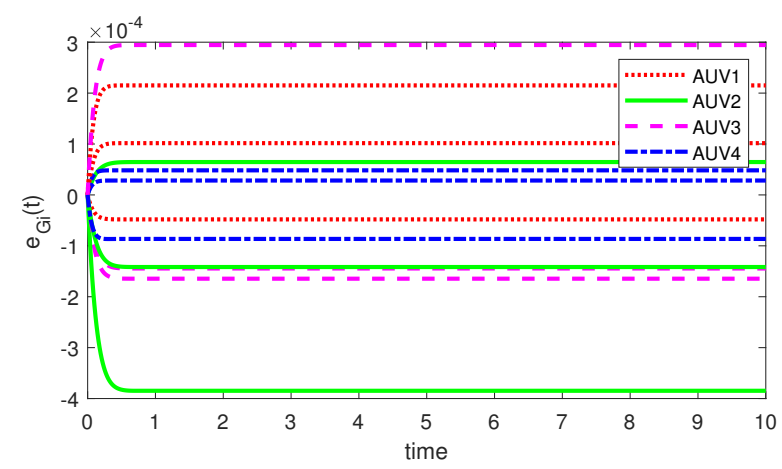

(d)

Fig. 8 The evolution of the parameter estimation errors under the adaptive laws (32). (a) The errors of $\hat{\mathbf{M}}_{i}$; (b) The errors of $\hat{\mathbf{C}}_{i}$; (c) The errors of $\hat{\mathbf{D}}_{i}$; (d) The errors of $\hat{\mathbf{g}}_{i}$. heterogeneous dynamics. We have proposed a two-layer distributed control strategy, where the upper layer provides a distributed finite-time observer for each AUV to estimate the state of the leader, and the lower layer offers a consensus tracking control algorithm based on a sliding mode method. Theoretical analysis showed that each follower AUV can track the leader in finite-time. When the parameters within the heterogenous AUV models are unknown, we have further introduced adaptive laws to estimate the parameters and thus improve the performance of the finite-time sliding mode control. In future, we will further consider the finite-time or fixed-time consensus control of AUVs with velocity-free measurements.

Acknowledgements This work was supported in part by the Sichuan Science and Technology Program under Grant 2020YFSY0012; in part by the National Natural Science Foundation of China under Grant 61473 061, Grant 71503206, and Grant 61104104; and in part by the Program for New Century Excellent Talents in University under Grant NCET13-0091

\section{Conflict of interest}

The authors declare that they have no conflict of interest.

\section{References}

1. Fossen, T.I.: Marine control systems: guidance, navigation and control of ships, rigs and underwater vehicles. Trondheim: Marine Cybernetics (2002)

2. Foresti, G.L.: Visual inspection of sea bottom structures by an autonomous underwater vehicle. IEEE Trans. Syst. Man. Cybern. Part B (Cybern.). 31(5), 691-705 (2001)

3. Gao, J., Proctor, A.A., Shi, Y., Bradley, C.: Hierarchical model predictive image-based visual servoing of underwater vehicles with adaptive neural network dynamic control. IEEE Trans. Cybern. 46(10), 2323-2334 (2016)

4. Cui, R., Yang, C., Li, Y., Sharma, S.: Adaptive neural network control of AUVs with control input nonlinearities using reinforcement learning. IEEE Trans. Syst. Man. Cybern.: Syst. 47(6), 1019-1029 (2017)

5. Cui, R., Ge, S.S., How, B.V.E., Choo, Y.S.: Leader-follower formation control of under actuated autonomous underwater vehicles. Ocean Eng. 37(17), 1491-1502 (2010)

6. Hou, S.P., Cheah, C.C.: Can a simple control scheme work for a formation control of multiple autonomous underwater vehicles? IEEE Trans. Control Syst. Tech. 19(5), 1090-1101 (2011)

7. Yang, E., Gu, D.: Nonlinear formation-keeping and mooring control of multiple autonomous underwater vehicles. IEEE/ASME Trans. Mech. 12(2), 164-178 (2007)

8. Li, J., Du, J., Chang, W.: Robust time-varying formation control for underactuated autonomous underwater vehicles with disturbances under input saturation. Ocean Eng. 179(1), 180-188 (2019)

9. Duan, K., Fong, S., Chen, C.L.P.: Multilayer neural networksbased control of underwater vehicles with uncertain dynamics and disturbances. Nonlinear Dyn. 100(4), 3555-3573 (2020)

10. Yuan, C., Licht, S., He, H.: Formation learning control of multiple autonomous underwater vehicles with heterogeneous nonlinear uncertain dynamics. IEEE Trans. Cybern. 48(10), 2920-2934 (2018) 
11. Elhaki O., Khoshnam, S.: Neural network-based target tracking control of underactuated autonomous underwater vehicles with a prescribed performance. Ocean Eng. 167(1) 239-256 (2018)

12. Peng, Z., Wang, D., Li, T., Han, M.: Output-feedback cooperative formation maneuvering of autonomous surface vehicles with connectivity preservation and collision avoidance. IEEE Trans. Cybern. 50(6), 2527-2535 (2020)

13. Yu, C., Xiang, X., Philip, P.A., Zhang, Q.: Guidance-error-based robust fuzzy adaptive control for bottom following of a flightstyle AUV with saturated actuator dynamics. IEEE Trans. Cybern. 50(5), 1887-1899 (2020)

14. Taha, E., Mohamed, Z., Kamal, Y.T.: Trajectory tracking sliding mode control of underactuated AUVs. Nonlinear Dyn. 84(5), 1079-1091 (2016)

15. Taha, E., Mohamed, Z., Kamal, Y.T.: Terminal sliding mode control for the trajectory tracking of underactuated autonomous underwater vehicles. Ocean Eng. 129(1), 613-625 (2017)

16. Cui, R., Zhang, X., Cui, D.: Adaptive sliding-mode attitude control for autonomous underwater vehicles with input nonlinearities. Ocean Eng. 123(1), 45-54 (2016)

17. Wang, Y., Zhang, M., Wilson, P.A., Liu, X.: Adaptive neural networkbased backstepping fault tolerant control for underwater vehicles with thruster fault. Ocean Eng. 110, 15-24 (2015)

18. Zakeri, E., Farahat, S., Moezi, S.A., Zare, A.: Robust sliding mode control of a mini unmanned underwater vehicle equipped with a new arrangement of water jet propulsions: simulation and experimental study. Appl. Ocean Res. 59, 521-542 (2016)

19. Li, X., Zhu, D.: Formation control of a group of AUVs using adaptive high order sliding mode controller. in OCEANS 2016Shanghai, Shanghai, 1-6 (2016)

20. Sun, Z., Zhang, G., Lu, Y., Zhang, W.: Leader-follower formation control of underactuated surface vehicles based on sliding mode control and parameter estimation. ISA Trans. 72, 15-24 (2018)

21. Yang, X., Yan, J., Hua, C., Guan, X.: Trajectory tracking control of autonomous underwater vehicle with unknown parameters and external disturbances. IEEE Trans. Syst. Man. Cybern.: Syst. 51(2), 1054-1063 (2021)

22. Wang, J., Wang, C., Wei, Y., Zhang, C.: Sliding mode based neural adaptive formation control of underactuated AUVs with leaderfollower strategy. Appl. Ocean Res. 94, 101971 (2020)

23. Liu, S., Liu, Y., Wang, N.: Nonlinear disturbance observer-based backstepping finite-time sliding mode tracking control of underwater vehicles with system uncertainties and external disturbances. Nonlinear Dyn. 88(1), 465-476 (2017)

24. Ali, N., Tawiah, I., Zhang, W.: Finite-time extended state observer based nonsingular fast terminal sliding mode control of autonomous underwater vehicles. Ocean Eng. 218(15), 108179 (2020)

25. Gao, Z., Guo, G.: Command filtered finite-time formation tracking control of AUVs with unknown control directions. IET Control Theory Appl. 14(13), 1744-1752 (2020)

26. Qin, H., Chen, H., Sun, Y., Chen, L.: Distributed finite-time faulttolerant containment control for multiple ocean bottom flying node systems with error constraints. Ocean Eng. 189, 106341 (2019)

27. Li, X., Luo, X., Wang, J., Guan, X.: Finite-time consensus of nonlinear multi-agent system with prescribed performance. Nonlinear Dyn. 91(4), 2397-2409 (2018)

28. Li, S., Wang, X.: Finite-time consensus and collision avoidance control algorithms for multiple AUVs. Automatica (Oxford). 49(11), 3359-3367 (2013)

29. Hong, Y., Hu, J., Gao, L.: Tracking control for multi-agent consensus with an active leader and variable topology. Automatica (Oxford). 42(7), 1177-1182 (2006)

30. Bhat, S.P., Bernstein, D.S.: Geometric homogeneity with applications to finite-time stability. Math. Control, Signals. Syst. 17(2), 101-127 (2005)
31. Wang, H., Chen, B., Lin, C., Sun, Y., Wang, F.: Adaptive finitetime control for a class of uncertain high-order nonlinear systems based on fuzzy approximation. IET Control Theory Appl. 11(5), 677-684 (2017)

32. Kim, Y.I., Yang, X.: Generalizations and refinements of Hölder's inequality. Appl. Math. Let. 25(7), 1094-1097 (2012)

33. Hardy, G., Littlewood, J., Polya, G.: Inequalities. Cambridge University Press. Cambridge, U.K. (1952) 
Figures

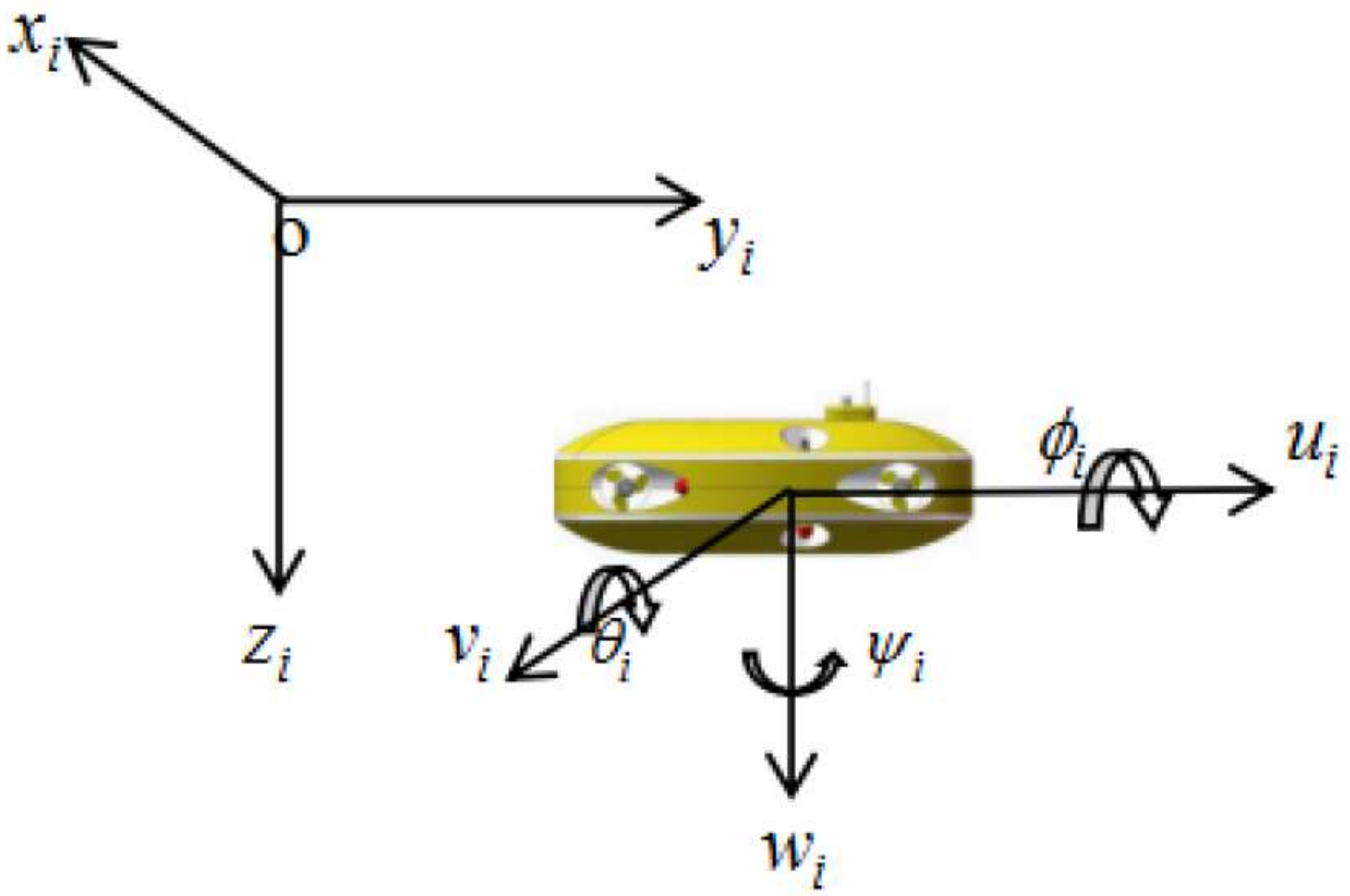

Figure 1

The reference frames of AUV. 


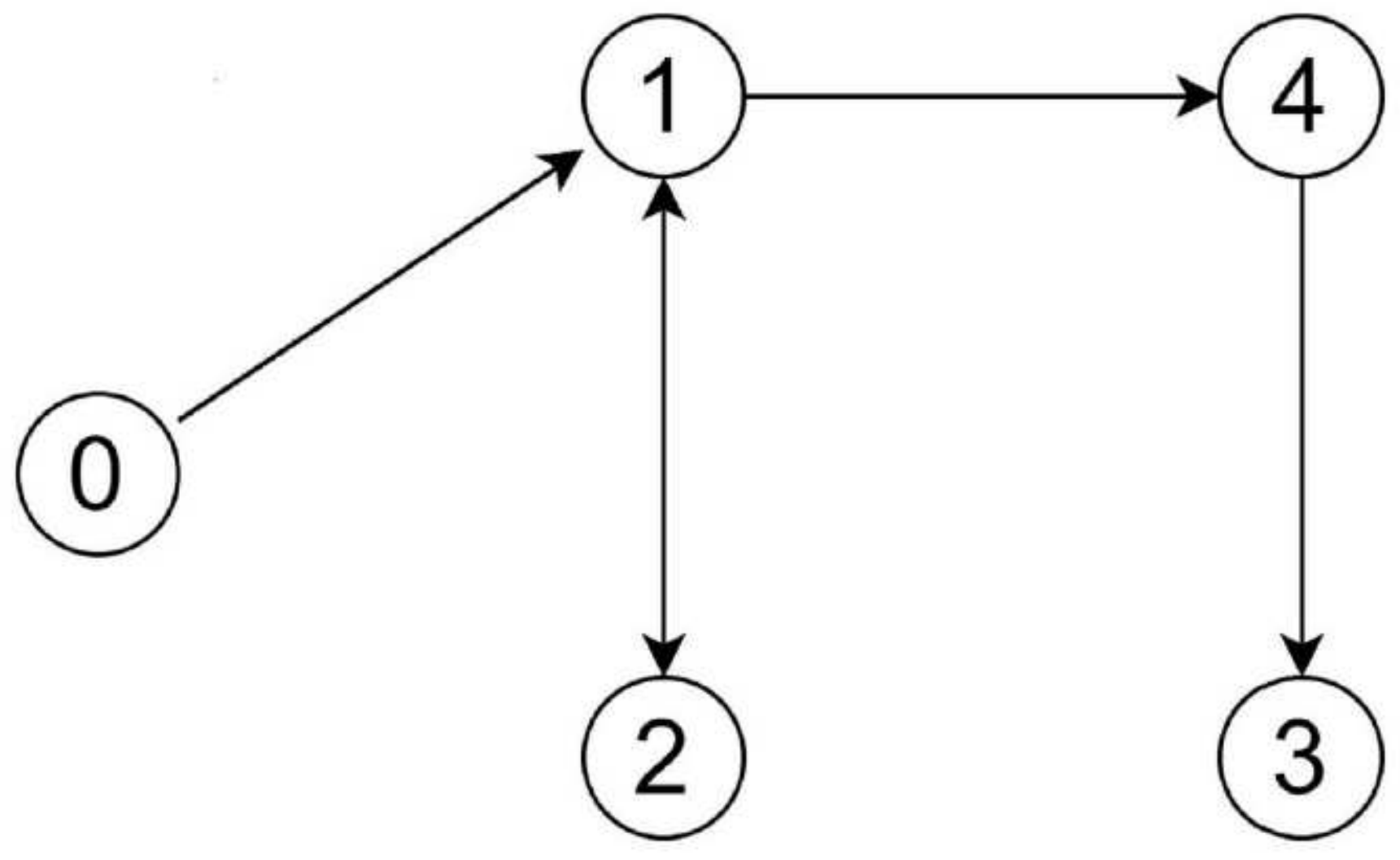

Figure 2

Interconnection network topology 


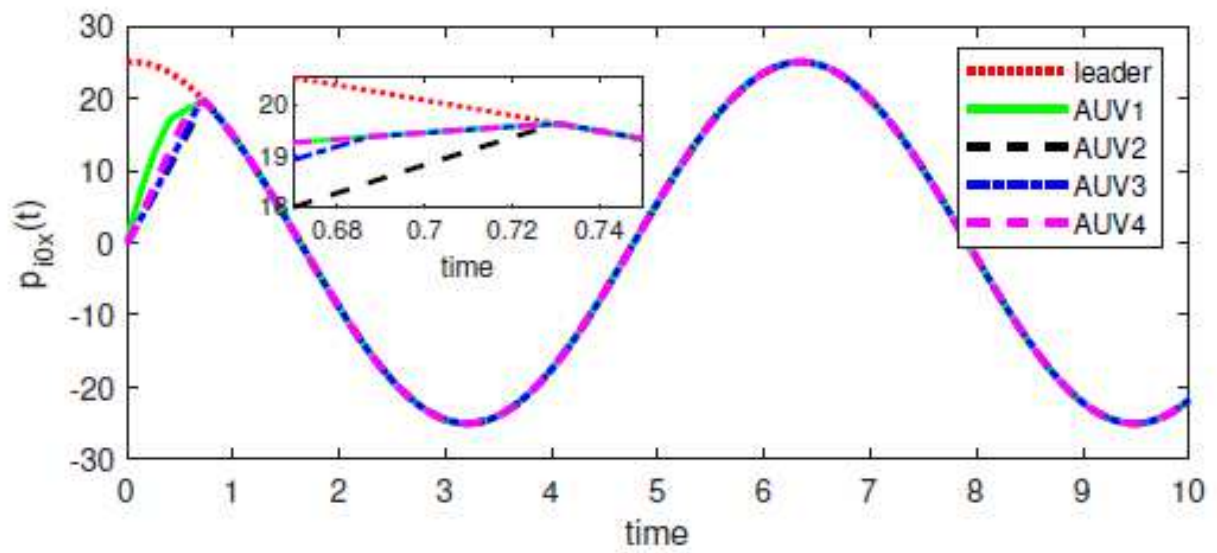

(a)

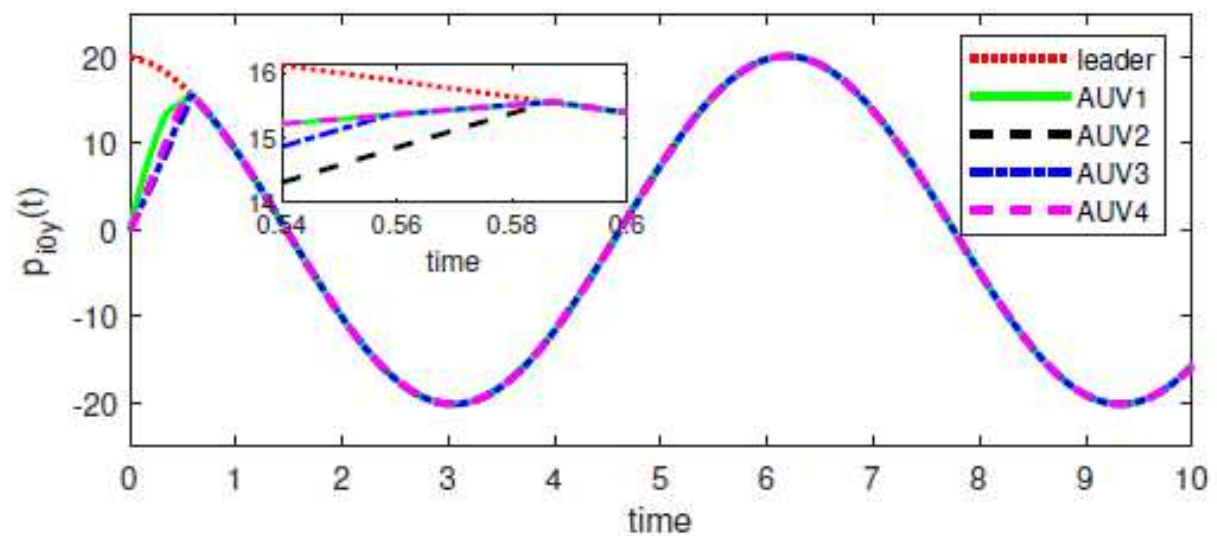

(b)

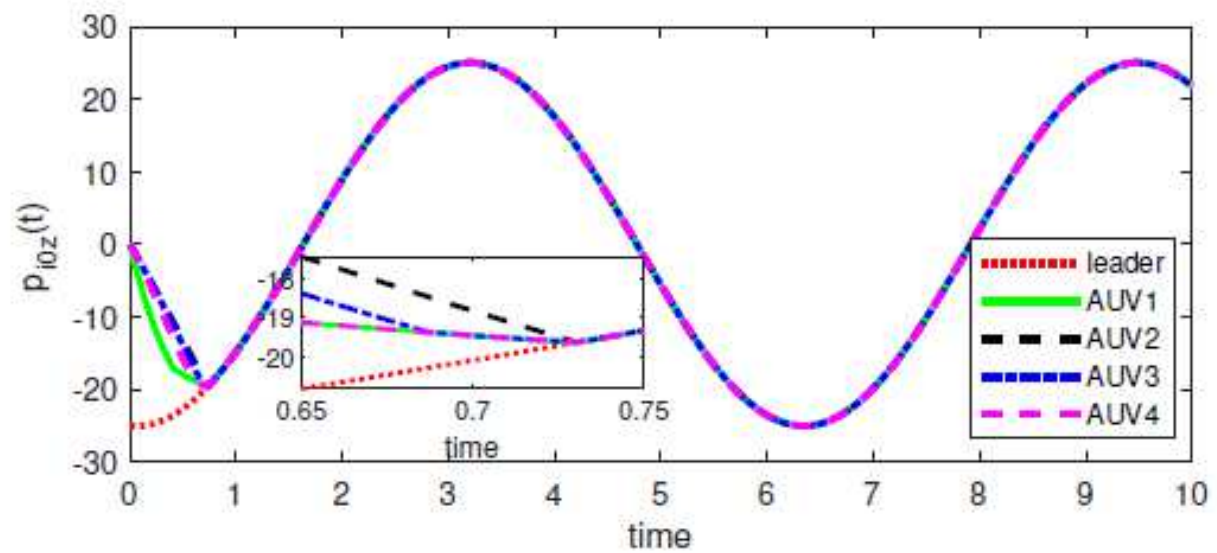

(c)

\section{Figure 3}

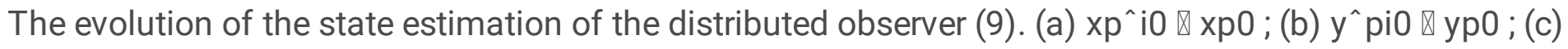
z^pi0 $\otimes z p 0$. 


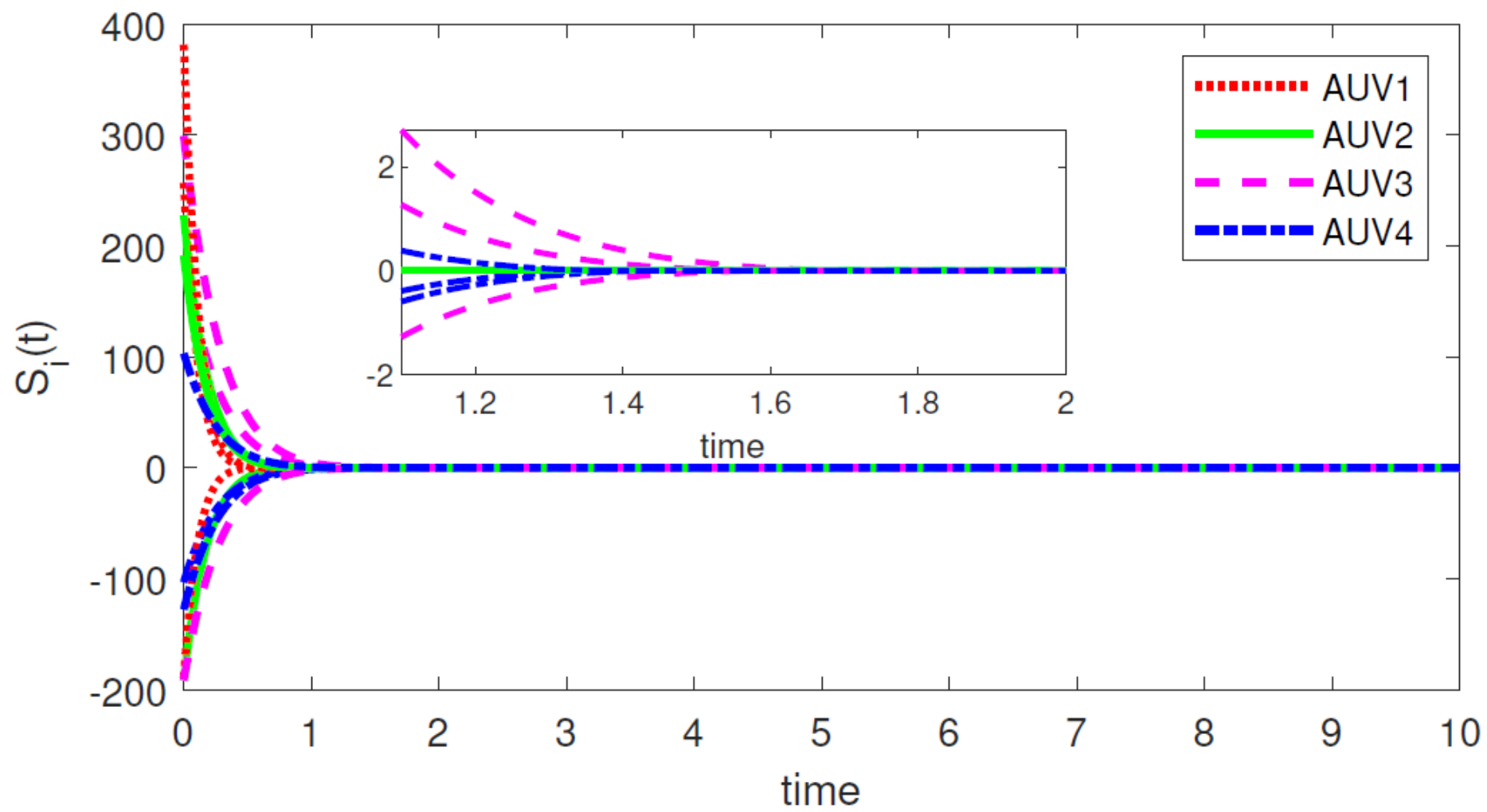

Figure 4

The evolution of the sliding mode surface (19). 


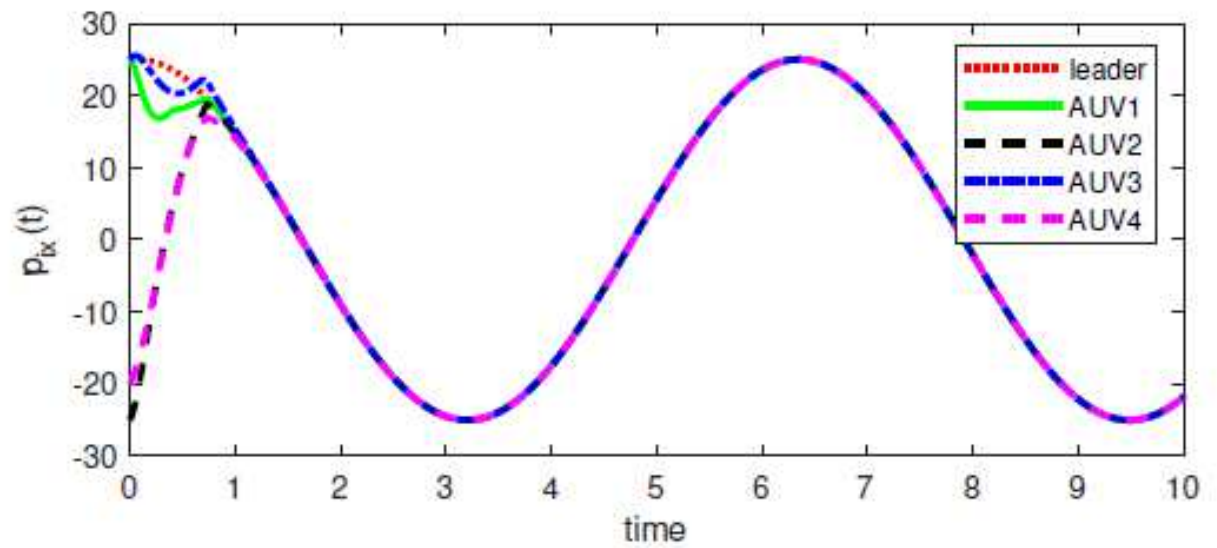

(a)

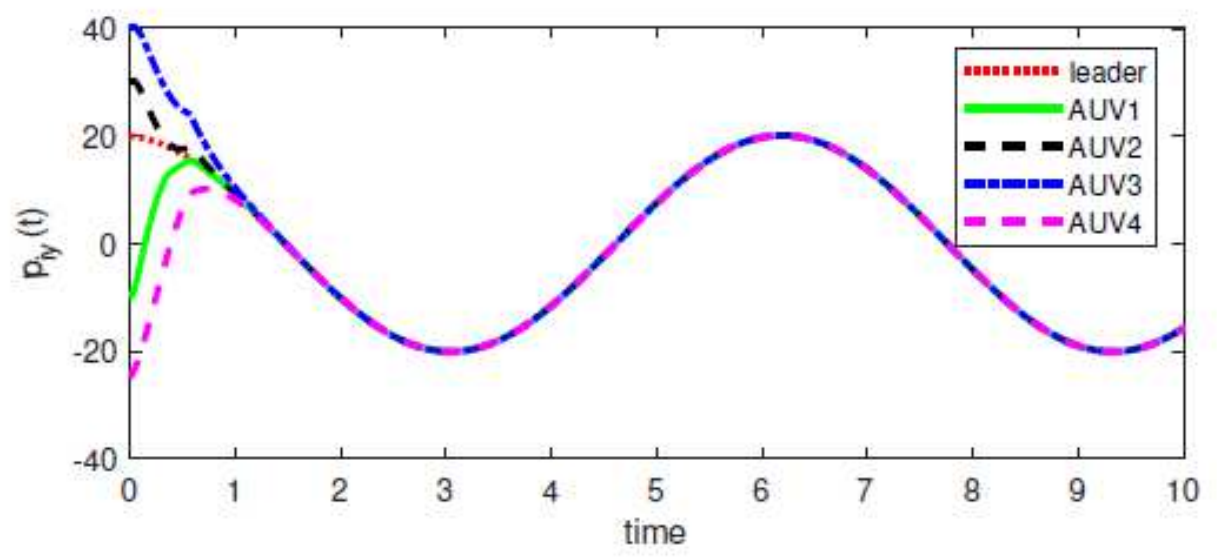

(b)

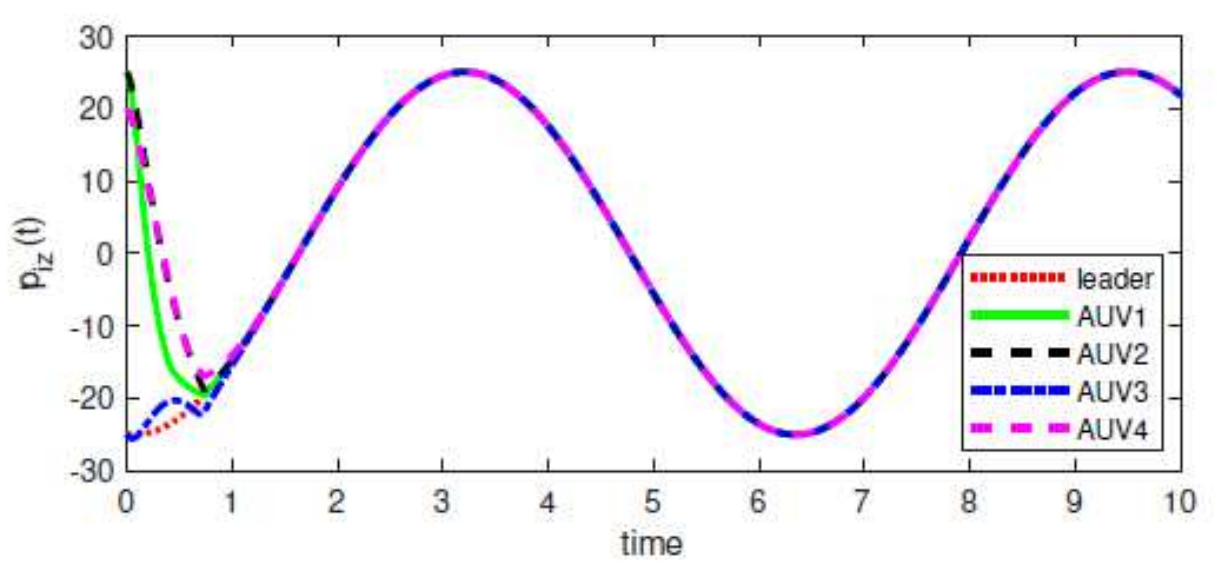

(c)

\section{Figure 5}

The position evolution under the control strategy (18). (a) xi $\otimes x 0$; (b) yi $\otimes y 0$; (c) zi $\otimes z 0$. 


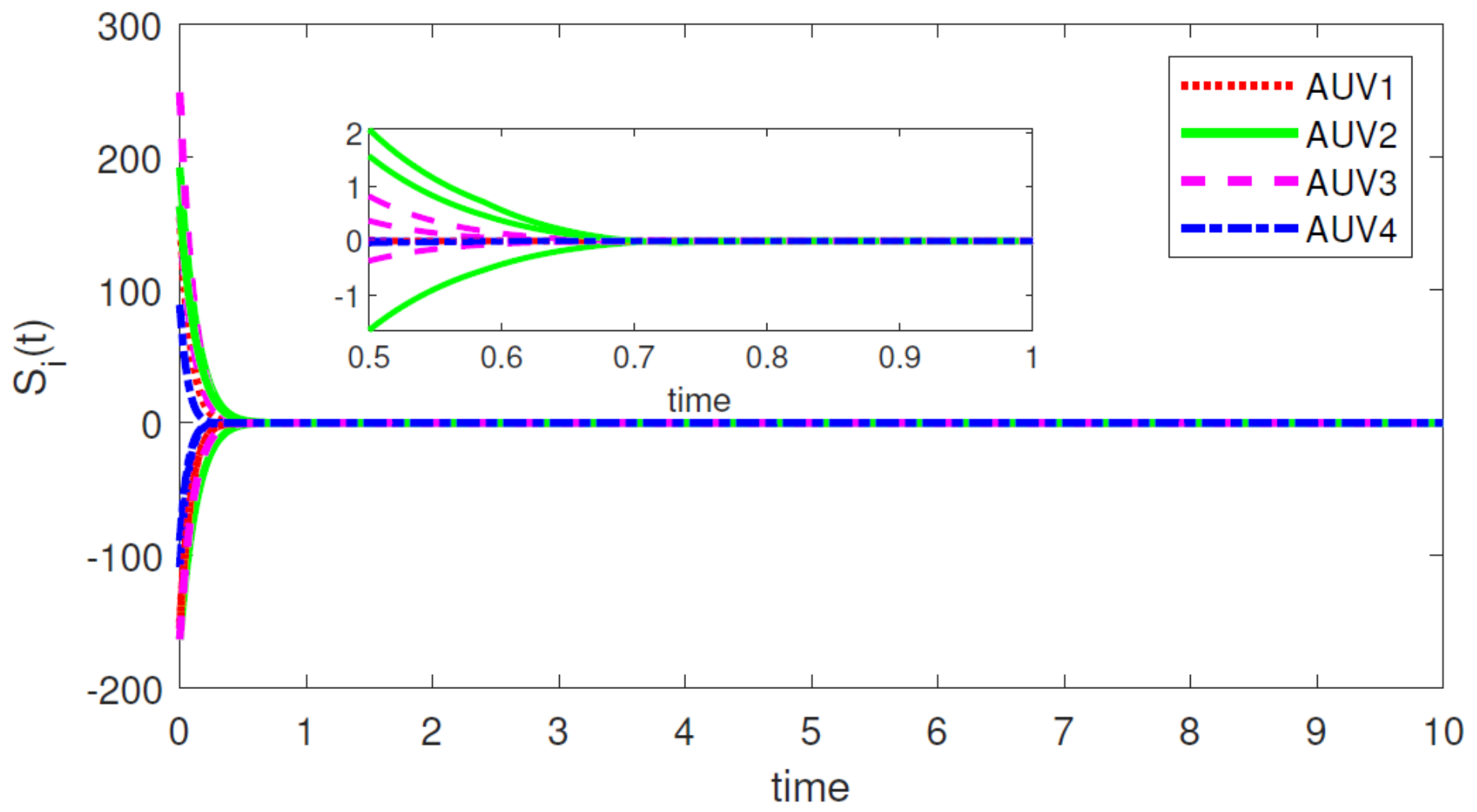

Figure 6

The evolution of the adaptive sliding mode surface under the controller (31). 


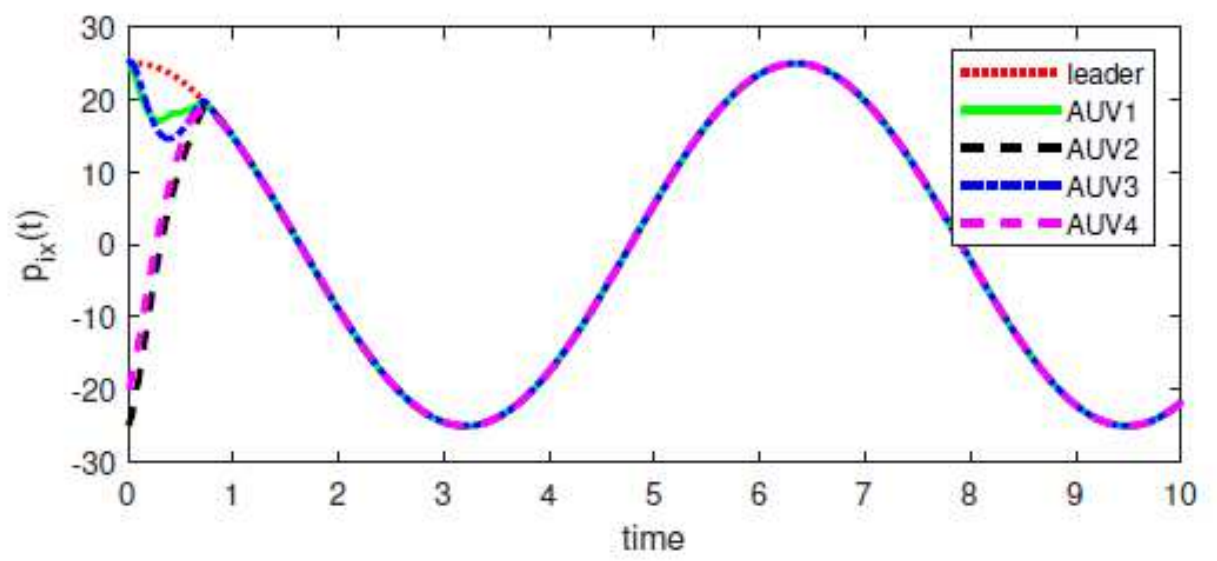

(a)

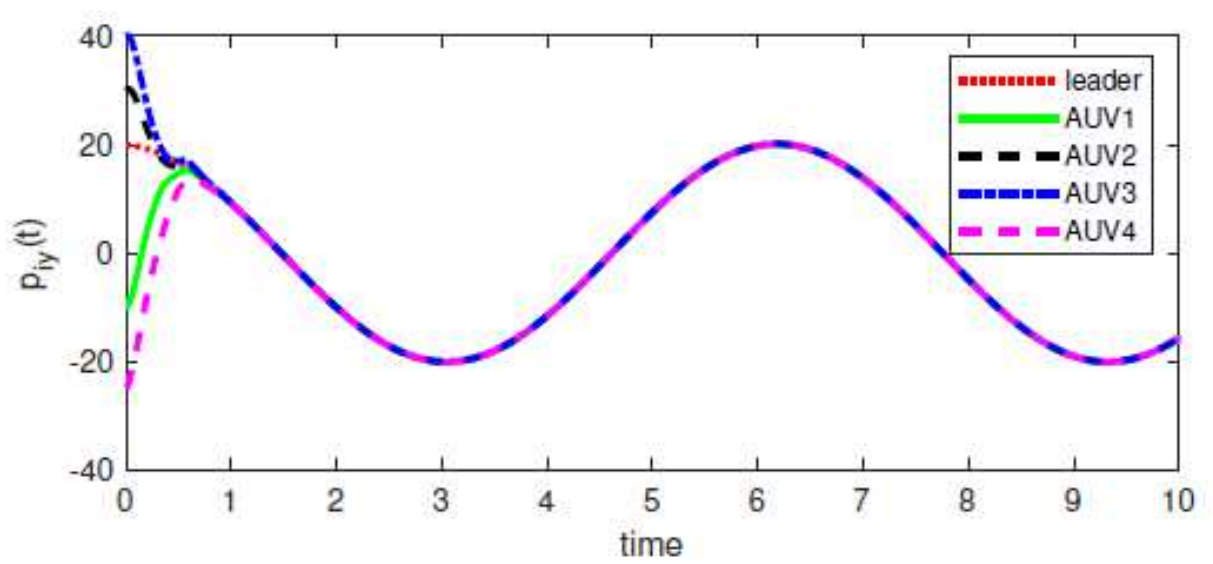

(b)

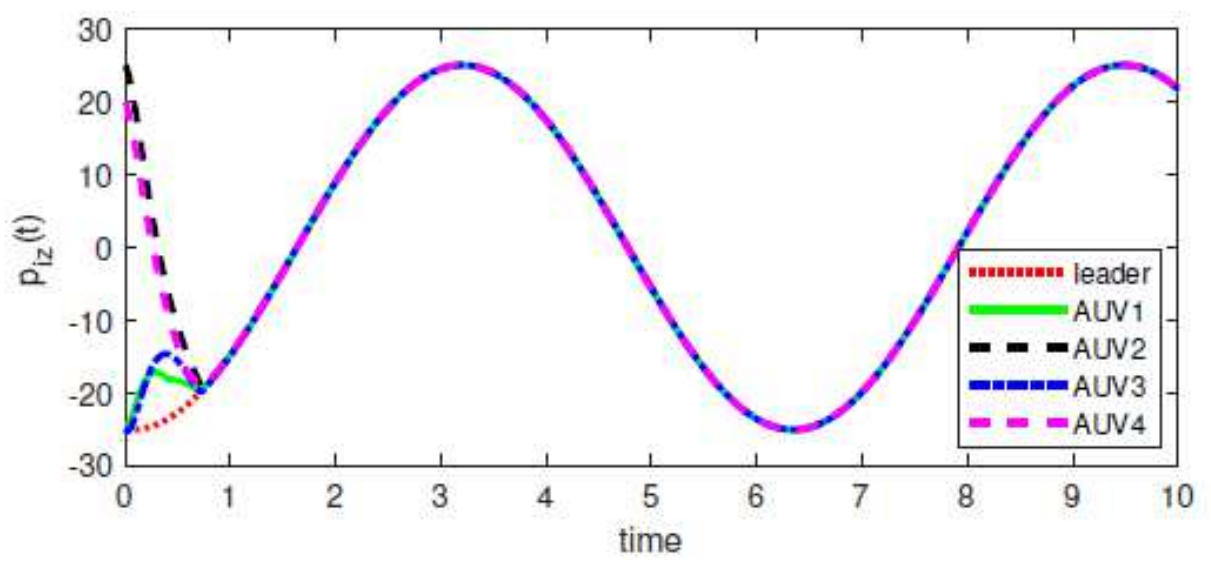

(c)

\section{Figure 7}

The position evolution under the adaptive sliding mode control (31). (a) xi $\nabla x 0$; (b) yi $\nabla y 0$; (c) zi $\nabla z 0$. 


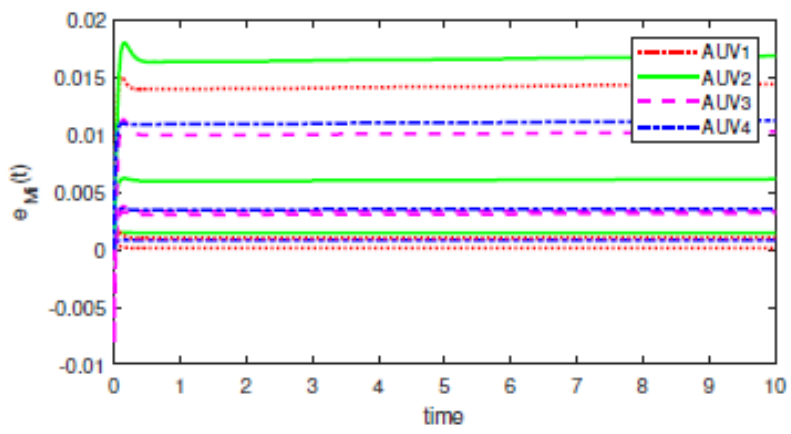

(a)

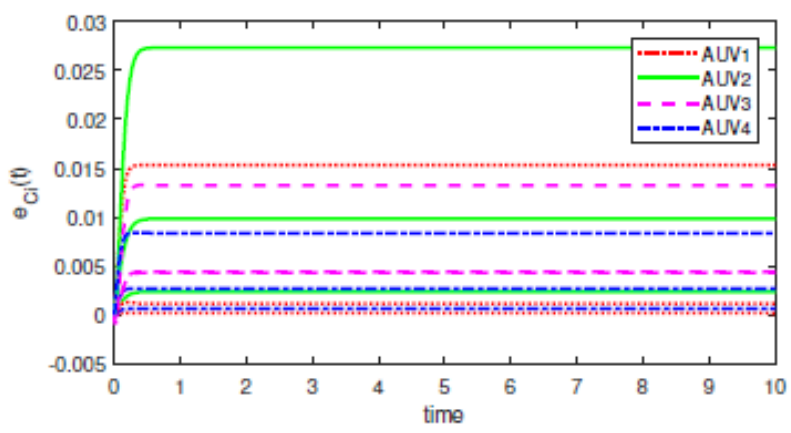

(b)

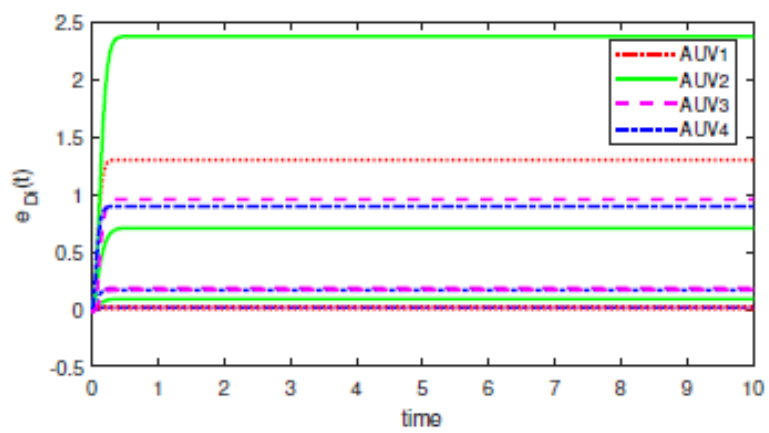

(c)

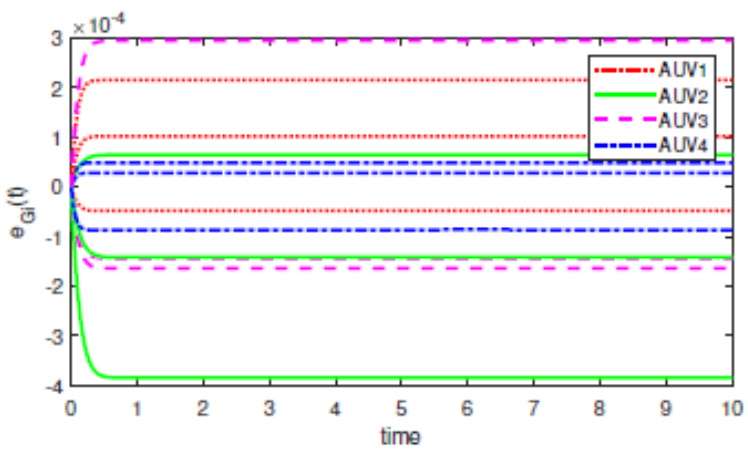

(d)

\section{Figure 8}

The evolution of the parameter estimation errors under the adap-tive laws (32). (a) The errors of ${ }^{\wedge} \mathrm{Mi}$; (b) The errors of ^ $\mathrm{Ci}$; (c) The errors of ^ $\mathrm{Di}$; (d) The errors of ^ $\mathrm{gi}$. 